\title{
The response of fabric variations to simple shear and migration recrystallization
}

\author{
Joseph H. KENNEDY,, ${ }^{1,2}$ Erin C. PETTIT ${ }^{3}$ \\ ${ }^{1}$ Computational Earth Sciences Group, Oak Ridge National Laboratory, Oak Ridge, TN, USA \\ ${ }^{2}$ Department of Physics, University of Alaska Fairbanks, Fairbanks, AK, USA \\ ${ }^{3}$ Department of Geosciences, University of Alaska Fairbanks, Fairbanks, AK, USA \\ Correspondence: Joseph H. Kennedy <kennedyjh@ornl.gov>
}

\begin{abstract}
The observable microstructures in ice are the result of many dynamic and competing processes. These processes are influenced by climate variables in the firn. Layers deposited in different climate regimes may show variations in fabric which can persist deep into the ice sheet; fabric may 'remember' these past climate regimes. We model the evolution of fabric variations below the firn-ice transition and show that the addition of shear to compressive-stress regimes preserves the modeled fabric variations longer than compression-only regimes, because shear drives a positive feedback between crystal rotation and deformation. Even without shear, the modeled ice retains memory of the fabric variation for $\sim 200 \mathrm{ka}$ in typical polar ice-sheet conditions. Our model shows that temperature affects how long the fabric variation is preserved, but only affects the strain-integrated fabric evolution profile when comparing results straddling the thermal-activation-energy threshold $\left(\sim-10^{\circ} \mathrm{C}\right)$. Even at high temperatures, migration recrystallization does not eliminate the modeled fabric's memory under most conditions. High levels of nearest-neighbor interactions will, however, eliminate the modeled fabric's memory more quickly than low levels of nearest-neighbor interactions. Ultimately, our model predicts that fabrics will retain memory of past climatic variations when subject to a wide variety of conditions found in polar ice sheets.
\end{abstract}

KEYWORDS: anisotropic ice, climate change, ice crystal studies, ice rheology, recrystallization

\section{DISCLAIMER}

Oak Ridge National Laboratory is managed by UT-Battelle, LLC. This submission was written by the authors acting in their own independent capacity and not on behalf of UTBatelle, LLC, or its affiliates or successors.

\section{INTRODUCTION}

Observations show that the microstructure of ice records aspects of climate history (e.g. Paterson, 1991; Gow and others, 1997; Durand and others, 2007; Pettit and others, 2011). However, understanding if and how the climate 'memory' is preserved and evolved through time is challenging (Kennedy and others, 2013). Paterson (1991) showed that ice-age ice typically consists of smaller grains and stronger fabric (statistically preferred orientation of the ice crystal lattice) than Holocene ice, providing the first hint of a connection between paleoclimate and microstructure in an ice sheet. More recently, both thin-section data and sonic-velocity data from Dome C, East Antarctica, show an abrupt transition in the ice rheology at $1750 \mathrm{~m}$ depth, which corresponds to a transition between the warm MIS-5 (marine isotope stage 5 ) and the cool MIS-6 $\sim 150 \mathrm{ka}$ ago (Durand and others, 2007; Gusmeroli and others, 2012). Many microstructural features (e.g. dust particles, grain size, fabric) correlate with climate history (e.g. Durand and others, 2006a). This correlation is caused by a number of interdependent microstructural processes (e.g. Alley, 1992; Faria and others, 2014b).

Classically, three main processes account for the observed grain and fabric structure throughout the depth of the ice sheet (e.g. Alley and others, 1986; Alley, 1992; De La
Chapelle and others, 1998; Cuffey and Paterson, 2010). Normal grain growth is the temperature-controlled coarsening of grains through grain-boundary migration, in order to reduce the total grain-boundary energy stored in the ice. Large grains consume small grains in such a way that the average grain cross-sectional area increases linearly with time (Alley and others, 1986). This process is considered to be active throughout the depth of the ice sheet, but is counterbalanced by polygonization at intermediate depths of the ice sheet, where a steady grain-size profile is observed (Alley, 1992). Strain energy builds in a grain during deformation, and bending or twisting stresses cause dislocations to form a 'wall', which eventually divides (polygonizes) the grain and causes a small misorientation between the grains' crystal lattices. At the greatest depths of an ice sheet, where the temperature is $>-10^{\circ} \mathrm{C}$, migration recrystallization becomes active. Here temperatures are great enough for grain boundaries to move easily, driven by differences in stored strain energy (Alley, 1992). With high enough stored strain energy, it becomes energetically favorable to nucleate a new strain-free grain, which rapidly migrates through neighboring grains and has an orientation that is dependent on the applied stress (Duval and Castelnau, 1995). With sufficiently high rates of migration recrystallization, an inverse power-law relationship between grain size and stress forms (Jacka and Jun, 1994) and an entirely new fabric structure results. Because fabric and grain structure are a result of recrystallization processes, conventional wisdom is that fabric will not maintain any past climate information.

Although the classic three-process model, or 'tripartite paradigm', has successfully described the average grain size and fabric in ice cores, observations in the last decade have 
led to new perspectives on these processes (e.g. Faria and others, 2014a,b; cf. De La Chapelle and others, 1998). Techniques that allow observations on the scale of micrometers or smaller (e.g. Kipfstuhl and others, 2006; Obbard and others, 2006; Weikusat and others, 2011) are now common, whereas classical observations typically were on the scale of millimeters or larger (Faria and others, 2014a). Some observations from these high-resolution techniques cannot be accounted for under the tripartite paradigm if it is considered a description of the actual microstructural physics. For example, in Dronning Maud Land, Antarctica, the firn grain-boundary structure appears to have been dominated by migration recrystallization during the firn-ice transition, even though it is much colder (annual mean temperature $-46^{\circ} \mathrm{C}$ ) than where migration recrystallization is considered to be a dominant process $\left(\gtrsim-10^{\circ} \mathrm{C}\right.$; Kipfstuhl and others, 2009). However, due to its success in describing the microstructure observed in ice (summarized by Faria and others, 2014a,b), the tripartite paradigm provides a robust phenomenological model of the average grain size and especially the fabric evolution (as discussed in the next section), despite its imperfections.

Observations of more recrystallization in the firn and ice of an ice sheet than expected under the tripartite paradigm (e.g. Kipfstuhl and others, 2009) would seem to reinforce the conventional idea that fabric cannot preserve a memory of past climate. Indeed, the memory is unlikely to be stored directly in the grain structure or fabric strength of a particular layer of ice, as they are continually evolving. The relative differences in the fabric between layers, however, can be preserved under certain conditions (Kennedy and others, 2013).

Because the microstructural processes active in the firn are sensitive to climatic variables (Alley and others, 1990), layers of firn experiencing different climate regimes may have observable variations in the fabric which can be preserved. For example, in polar regions, vapor deposition is the primary method of grain growth in the upper firn and it is anisotropic: deposition will favor either the basal or prism faces of the ice-crystalline lattice, depending on the temperature (Nelson and Knight, 1998). This process causes grains with the preferred face parallel to the vapor-pressure gradient to grow more than grains in a less favorable orientation. Because these grains grow at the expense of other grains (Colbeck, 1983), the well-oriented grains are more likely to remain (Carns and others (2010) are developing a model to explore this process). Variations in texture and fabric in the firn may reflect variations in temperature and vapor-pressure gradients (Adams and Miller, 2003). While measuring fabric in firn is difficult, several studies have reported non-isotropic fabric measurements from firn (DiPrinzio and others, 2005; Fujita and others, 2009; Montagnat and others, 2012). Montagnat and others (2012) found that using non-isotropic initial fabrics was required during simulations of the fabric evolution in Talos Dome, East Antarctica, for a good quantitative match to observed fabrics. Therefore, fabric variations may arise from climatic variations and these variations may be present beneath the firn-ice transition. Fabric variations then may preserve memory of past climate, as long as the fabric variation is observable. (A loose analogy can be drawn to the familiar climate proxy, $\delta^{18} \mathrm{O}$; it is the variation in $\delta^{18} \mathrm{O}$, not the particular amount of the oxygen isotope present, that allows us to extract a temperature history.)
Here, we ask how long a subtle fabric variation (of any origin), just below the firn-ice transition, can be preserved within an ice sheet. Kennedy and others (2013) used a model based on the tripartite paradigm to show that a subtle variation in fabric can persist throughout the depth of an ice sheet when the ice is in a vertical uniaxial-compression or pure-shear regime and experiences polygonization events typical of ice divides. We build on this work to show that it is possible to preserve a subtle variation in fabric in a simple-shear stress regime and subject to migration recrystallization. Ice flows dominantly by simple shear on the flank of an ice sheet, while ice at the divide may experience some simple shear, especially in the case of divide migration. We also show that for any of the modeled stresses or temperatures, migration recrystallization does not 'erase' the fabric variation. Together, the combination of uniaxial compression, pure shear, simple shear, polygonization and migration recrystallization account for the dominant processes within an ice sheet that affect the fabric evolution.

\section{FABRIC}

A sample of ice can display a statistically preferred orientation of its crystal lattices called fabric. The statistical measure of this preference is often reported as eigenvalues and eigenvectors of the (volume-weighted) average orientation tensor of the sample. The grain volume can be determined from the measurement of the two-dimensional grain area in a cross section of the sample (Woodcock, 1977; Gagliardini and others, 2004).

The orientation tensor is calculated from a sample of $N$ grains:

$$
\boldsymbol{A}=\sum_{n=1}^{N} \overrightarrow{f_{n}} \overrightarrow{\mathrm{C}_{n}} \otimes \overrightarrow{\mathrm{C}_{n}},
$$

where $f_{n}$ is the estimate of the grain's volume fraction, $\overrightarrow{C_{n}}$ is a unit vector describing the grain's $c$-axis orientation and $\otimes$ is the vector direct (outer) product. The eigenvalues, $e_{i}$, for $i=1,2,3$, of $\boldsymbol{A}$ then represent the spatial distribution of the orientations, and how tightly the crystals are aligned to the eigenvectors, $\vec{v}_{i}$. The eigenvalues are labeled in descending order $\left(e_{1}>e_{2}>e_{3}\right)$ and sum to unity $\left(e_{1}+e_{2}+e_{3}=1\right)$. For a single-maximum fabric, the statistically preferred orientation is the first eigenvector, $\overrightarrow{v_{1}}$. The first eigenvalue, $e_{1}$, measures the fabric strength. Fabrics typically strengthen throughout the depth of an ice sheet in response to stressinduced velocity gradients driving grain rotation in the ice (e.g. Paterson, 1991; Arnaud and others, 2000; DiPrinzio and others, 2005; Durand and others, 2007; Gow and Meese, 2007).

Microstructural processes further influence the volume orientation of ice and can significantly impact the fabric statistics. The three that affect lattice orientation are rotation recrystallization (RRX), where new grain boundaries are formed through the progressive rotation and migration of subgrain boundaries (of which polygonization is a special case), and strain-induced boundary migration (SIBM; also called migration recrystallization) from old/existing grains (SIBM-O) and from nucleation of new grains (SIBM-N) (see Faria and others, 2014b, appendix A). Of these three, only SIMB-N is known to affect the fabric strength significantly. RRX produces new grains, of differing size, with small lattice misorientations $\left(<10^{\circ}\right.$; Alley and others, 1995), but the 

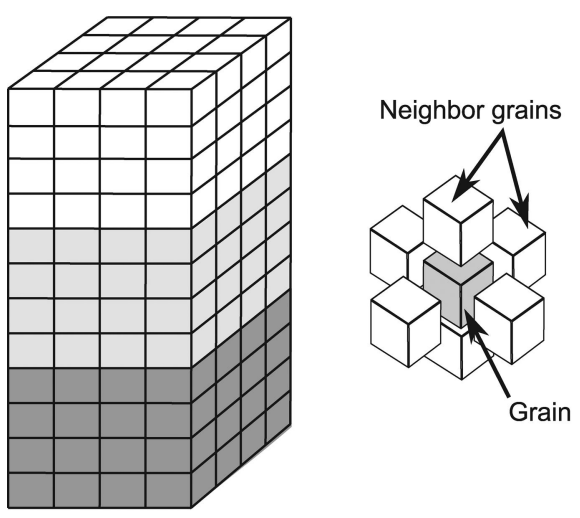

Fig. 1. The polycrystal structure. Left: An example of a polycrystalline cuboid with three distinct fabric layers, where each color represents a different fabric. Each small cube indicates one grain and each layer has $4 \times 4 \times 4=64$ grains. The three-layered cuboid in our model has $20 \times 20 \times 20=8000$ grains in each layer. Right: An illustration of the grain packing where each grain (gray) has six neighboring grains (white).

volume average of these new grains, and what is left of the old grain, will closely match the average before RRX, resulting in a small weakening of the fabric. SIBM-O, in a statistical sense, is erasing the orientation of a bit of ice and replacing it with an orientation drawn from the same parent distribution of grains. Given a large number of grains, this will not affect the overall fabric statistics as long as there is not a preference to recrystallize grains in a certain orientation and the total population of grains remains large. Due to the large amount of strain heterogeneity in deforming ice, there should not be an orientation preference for SIBM-O (Faria and others, 2014b).

SIBM-N, in contrast, nucleates new grains with a random orientation (Wilson and others, 2014). The fabrics that arise when SIBM-N dominates the fabric evolution are typically aligned for easy glide in the basal planes (the softest orientation) (Montagnat and others, 2009), indicating that the nucleated grains most likely to grow are the ones oriented for easy glide. The influence of SIBM-N on a fabric variation will then depend on the rates of SIBM-N in each layer, as well as the rates of grain rotation. As long as the rates of SIBM-N remain low, or there is a differing rate in the varying layers, SIBM-N will not immediately eliminate the fabric variation (as shown below).

Therefore, due to the way fabric is influenced by the microstructural processes, and the way it is measured, we expect fabric to be particularly amenable to preserving variations.

\section{THE MODEL}

We use the topological model developed by Kennedy and others (2013), based on the analytic flow law developed by Thorsteinsson (2001, 2002). This polycrystal model solves for fabric through time, while incorporating nearest-neighbor interactions (NNIs) and the tripartite parameterizations of normal grain growth, polygonization and migration recrystallization (SIBM-N). This model does not predict ice flow; therefore, it does not account for possible strain enhancements, such as impurity-enhanced ice flow (Paterson, 1991; Faria and others, 2009). Nor does it include the feedbacks between rheologically distinct layers that can

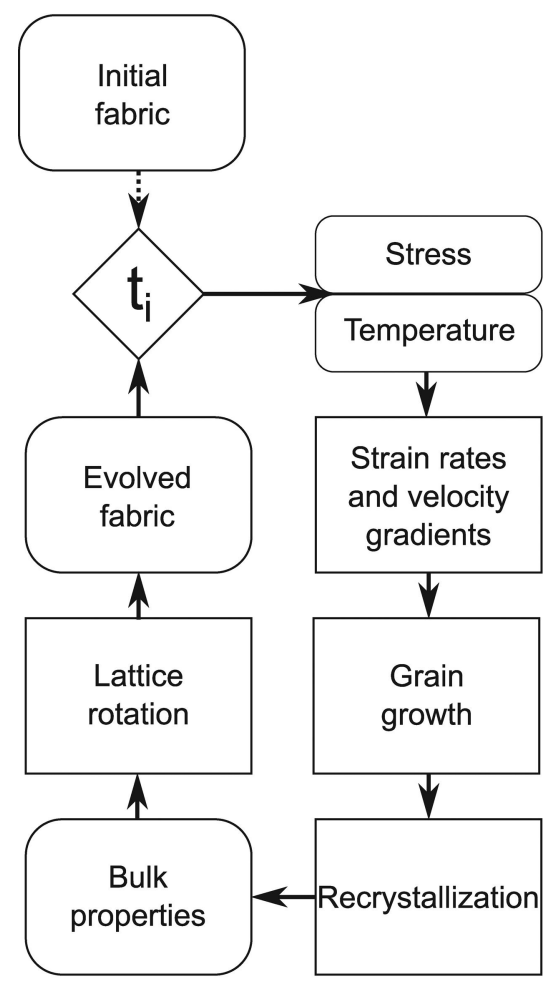

Fig. 2. Flow chart of the model. The model is initialized with fabric data, deviatoric stress and temperature. For each time step, strain rates and velocity gradients are calculated, dynamic recrystallization processes are applied to the fabric and then the grains are rotated to calculate new fabric data. The new fabric data together with new stresses and temperatures are fed back into the model to start the next time step.

lead to concentrated shearing on layers with crystals oriented to be soft in shear (Budd and Jacka, 1989; Durand and others, 2007; Pettit and others, 2007).

The model averages over a representative distribution of $N$ individual ice crystals to calculate the bulk response of the ice to stress. The crystals are arranged on a regular cuboidal grid (Fig. 1), where each crystal has six nearest neighbors. The crystals however, are considered to evolve independently of each other and are embedded in an ice matrix. The matrix accommodates crystal-boundary migration and acts as seeds for migration recrystallization (Thorsteinsson, 2002). In the case of NNIs, the resolved shear stress of the crystal is modified by a factor depending on the average orientation of the surrounding crystals.

The distribution of $N$ crystals can be divided into subdistributions with distinct fabrics. The evolution of each subdistribution can then be calculated and compared with the others or with the entire distribution through time.

Each crystal in the distribution has an associated orientation, $\overrightarrow{c_{n}}$, given by the co-latitude, $\theta_{n}$, and azimuth, $\phi_{n}$, as well as an associated spherical size of diameter $D_{n}$ and dislocation density $\rho_{n}$. The model accepts an initial crystal distribution, stress and temperature, then evolves the distribution through uniform steps in time or strain. Figure 2 outlines the model process. First, the initial crystal distribution is created and passed to the model. The model then applies a stress to the distribution and calculates the individual crystal strain rates and velocity gradients using the flow law developed by Thorsteinsson (2001, 2002). Next, it checks the recrystallization conditions (outlined 


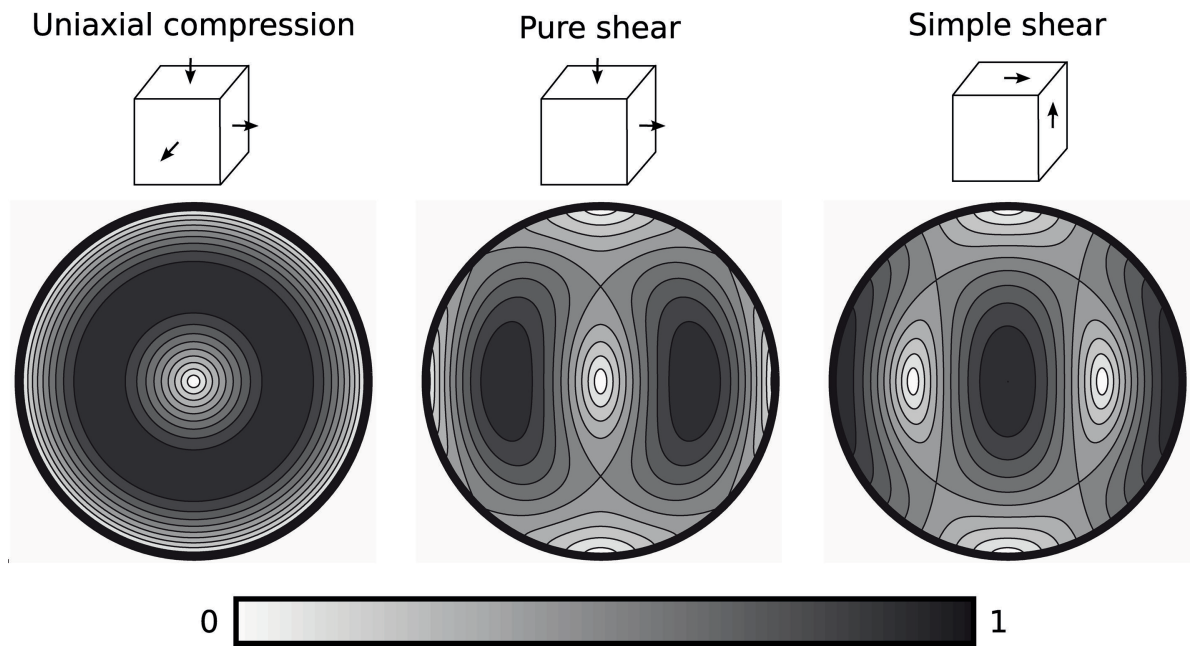

Fig. 3. Contoured Schmidt plots of the magnitude of the resolved shear stress, $\mathcal{T}$. $\mathcal{T}$ has been normalized by the maximum resolved shear stress $(\mathcal{T} / \max (\mathcal{T})$; Eqn (3)) for grains in the stress states of uniaxial compression, pure shear and simple shear (Eqns (18), (19) and (20), respectively).

below), calculates the bulk crystal properties and then rotates the crystals. After each time- or strain-step, the model outputs the new distribution of crystals, the bulk strain, and the number and type of recrystallization events. This new distribution of crystals is then fed back into the model for the next time step, along with the new stress and temperature. Because stress is an input for this model, stress must be determined outside of the model.

\subsection{Model physics}

Grains rotate due to gradients in velocity, which result from internal stresses experienced by the ice. For polycrystalline ice, these stresses lie somewhere between two end-member assumptions: uniform stress and uniform strain rates. In the uniform-stress assumption, each grain experiences the same stress as the surrounding grains. Because ice crystals are highly anisotropic, an individual grain's strain rate, therefore, depends on its lattice orientation. In the uniform-strainrate assumption, each grain has the same strain rate as the surrounding grains. The stress the grain experiences, then, depends on the grain's orientation. Although the true nature of ice is somewhere in between, the uniform-stress assumption is well adapted to describing polycrystalline ice, due to strong crystal anisotropy (Castelnau and others, 1996). Therefore, we apply a uniform stress to each grain in the distribution. Furthermore, we restrict the deformation of a grain to be along the slip systems in the basal plane, causing the grain to only respond to the components of stress that are parallel to the basal plane (termed the resolved shear stress, or RSS). The RSS, $\tau^{(s)}$, on a slip system, $(s)$, is

$$
\tau^{(s)}=\boldsymbol{S}^{(s)}: \boldsymbol{\sigma}^{\prime},
$$

where $\boldsymbol{S}^{(s)}$ is the Schmidt tensor, which describes the orientation of the grain's slip system, $\sigma^{\prime}$ is the deviatoric stress tensor for the stress applied to the fabric and $\boldsymbol{S}^{(s)}: \boldsymbol{\sigma}^{\prime}=S_{k l}^{(s)} \sigma_{k l}^{\prime}$ summing over repeated indexes. The magnitude of the RSS, $\mathcal{T}$, can then be calculated as

$$
\mathcal{T}=\left|\sum_{(s)} \tau^{(s)} \widehat{b}^{(s)}\right|
$$

where $\widehat{b}^{(s)}$ is the direction of the Burgers vector for the slip system. Schmidt plots of $\mathcal{T}$ for a variety of stress states are shown in Figure 3.

Using the analytic flow law (Thorsteinsson, 2001, 2002), the velocity gradient of a grain in response to a stress, $\boldsymbol{L}^{\mathrm{C}}$, is

$$
\boldsymbol{L}^{\mathrm{c}}=\beta \mathcal{A}(T) \sum_{(s)} \boldsymbol{S}^{(s)}\left|\mathcal{E}^{\mathrm{c}} \tau^{(s)}\right|^{n-1}\left(\mathcal{E}^{\mathrm{c}} \tau^{(s)}\right)
$$

where $\beta$ is an adjustable constant to control the isotropic ice softness, $\mathcal{A}(T)$ is the temperature-dependent flow parameter from Glen's flow law (Cuffey and Paterson, 2010, p. 72), $\mathcal{E}^{\mathrm{c}}$ is the local softness parameter due to NNIs, and $n$ is the exponent in Glen's flow law (Glen, 1955).

The flow parameter $\mathcal{A}(T)$ follows an Arrhenius relation with a switch of activation energy at a transition temperature, $T_{\star}=-10^{\circ} \mathrm{C}$. The relationship for $T$ in kelvin is

$$
\mathcal{A}(T)=\mathcal{A}_{\star} \exp \left(-\frac{Q_{V}^{ \pm}}{R}\left[\frac{1}{T}-\frac{1}{T_{\star}}\right]\right),
$$

where $\mathcal{A}_{\star}$ is a constant, $R$ is the universal gas constant and $Q_{v}^{ \pm}$is the activation energy for volume self-diffusion (Cuffey and Paterson, 2010, p. 72). $Q_{v}^{ \pm}=Q_{v}^{+}=115 \mathrm{~kJ} \mathrm{~mol}^{-1}$ for $T \geq T_{\star}$ and $Q_{v}^{ \pm}=Q_{v}^{-}=60 \mathrm{~kJ} \mathrm{~mol}^{-1}$ for $T<T_{\star}$ (Table 1$)$.

The local softness parameter, $\mathcal{E}^{\mathrm{C}}$, averages the magnitude of RSS the neighboring grains are experiencing, $\mathcal{T}^{i}$, relative to the magnitude of RSS the grain is experiencing, $\mathcal{T}^{0}$ :

$$
\mathcal{E}^{\mathrm{c}}=\frac{1}{\zeta+6 \xi}\left(\zeta+\xi \sum_{i=1}^{6} \frac{\mathcal{T}^{i}}{\mathcal{T}^{0}}\right),
$$

where $\zeta$ is the relative contribution of the center grain, and $\xi$ is the relative contribution of each neighbor (Fig. 1). Because the RSS, $\mathcal{T}^{0}$, can be zero, there is a specified cap for the maximum value of $\mathcal{E}^{\mathrm{c}}$. Setting $[\zeta, \xi]$ to $[1,0]$ in Eqn (6) is equivalent to the uniform-stress assumption with no NNIs. Changing the values of $[\zeta, \xi]$ modifies the uniform-stress assumption (toward the uniform-strain assumption) by redistributing the stress through explicit NNIs. Mild NNIs occur when $[\zeta, \xi]$ is set to $[6,1]$, such that the center grain contribution to $\mathcal{E}^{\mathrm{C}}$ is the same as the sum of the neighboring grains. Full NNIs occur when $[\zeta, \xi]$ is set to $[1,1]$, such that 
Table 1. Values of the parameters used in the model

\begin{tabular}{|c|c|c|c|}
\hline Parameter & Value & Equation & Source \\
\hline Flow law constant, $\beta$ & 630 & (4) & Thorsteinsson (2001) \\
\hline Flow law constant, $\mathcal{A}_{\star}$ & $3.5 \times 10^{-25} \mathrm{~Pa}^{-3} \mathrm{~s}^{-1}$ & (5) & Cuffey and Paterson (2010, p. 74) \\
\hline Activation energy for volume self-diffusion, $Q_{v}^{ \pm}$ & $\begin{array}{l}Q_{v}^{+}=115 \mathrm{~kJ} \mathrm{~mol}^{-1} \text { if } T \geq-10^{\circ} \mathrm{C} \\
Q_{v}^{-}=60 \mathrm{~kJ} \mathrm{~mol}^{-1} \text { if } T<-10^{\circ} \mathrm{C}\end{array}$ & (5) & Cuffey and Paterson (2010, p. 72-74) \\
\hline Initial grain diameter, $D_{0}$ & $1.5 \mathrm{~mm}$ & (10) & Benson (1962) \\
\hline Grain growth constant, $K_{0}$ & $8.2 \times 10^{-9} \mathrm{~m}^{2} \mathrm{~s}^{-1}$ & (11) & Alley and others (1986), Thorsteinsson (2002) \\
\hline Activation energy for grain-boundary self-diffusion, $Q_{b}^{ \pm}$ & $\begin{array}{l}Q_{\mathrm{b}}^{+}=81 \mathrm{~kJ} \mathrm{~mol}^{-1} \text { if } T \geq-10^{\circ} \mathrm{C} \\
Q_{\mathrm{b}}^{-}=42 \mathrm{~kJ} \mathrm{~mol}^{-1} \text { if } T<-10 \mathrm{C}\end{array}$ & $(11)$ & $\begin{array}{c}\text { Cuffey and Paterson (2010, p. 40) } \\
\text { Jacka and Jun (1994) }\end{array}$ \\
\hline Dislocation absorption constant, $\alpha$ & 1 & $(12)$ & $\begin{array}{c}\text { De La Chapelle and others (1998), Montagnat } \\
\text { and Duval (2000) }\end{array}$ \\
\hline Polygonization ratio, $\delta$ & 0.065 & & Thorsteinsson (2002) \\
\hline Polygonization orientation change, $\Delta \theta$ & $5^{\circ}$ & & Thorsteinsson (2002) \\
\hline Initial dislocation density, $\rho_{0}$ & $10^{10} \mathrm{~m}^{-2}$ & & De La Chapelle and others (1998) \\
\hline Dislocation energy constant, $\mu$ & 0.01 & (13) & $\begin{array}{l}\text { Mohamed and Bacroix (2000), Thorsteinsson } \\
\text { (2002) }\end{array}$ \\
\hline Dislocation strain field range, $R_{\mathrm{e}}$ & $1 / \sqrt{\rho}$ & (13) & Mohamed and Bacroix (2000) \\
\hline Grain-boundary energy, $\gamma_{\mathrm{g}}$ & $0.065 \mathrm{~J} \mathrm{~m}^{-2}$ & (14) & Ketcham and Hobbs (1969) \\
\hline
\end{tabular}

the center grain and individual neighbor grains all contribute equally to $\mathcal{E}^{\mathrm{c}}$.

Finally, the strain rate of a single grain is

$$
\dot{\boldsymbol{\epsilon}}^{\mathrm{C}}=\frac{1}{2}\left[\boldsymbol{L}^{\mathrm{C}}+\left(\boldsymbol{L}^{\mathrm{C}}\right)^{\mathrm{T}}\right]
$$

where $\left(\boldsymbol{L}^{\mathrm{C}}\right)^{\mathrm{T}}$ refers to the matrix transpose of $\boldsymbol{L}^{\mathrm{C}}$.

The bulk velocity gradient is calculated by averaging the single-crystal velocity gradients, and will be influenced more by larger crystals than smaller crystals. We calculate the volume of a crystal from its diameter, $D$, and use its volume fraction, $f$, as a statistical weight for the calculation of the bulk velocity gradient (Gödert and Hutter, 1998; Montagnat and others, 2014). Therefore, the modeled bulk velocity gradient is

$$
\boldsymbol{L}^{\boldsymbol{m}}=\sum_{n=1}^{N} f_{n} \boldsymbol{L}_{n^{\prime}}^{\mathrm{c}}
$$

where $N$ is the number of crystals in the representative distribution and

$$
f_{n}=\frac{D_{n}^{3}}{\sum_{m=1}^{N} D_{m}^{3}} .
$$

The modeled bulk strain rate is then

$$
\dot{\boldsymbol{\epsilon}}^{\mathrm{m}}=\frac{1}{2}\left[\boldsymbol{L}^{\boldsymbol{m}}+\left(\boldsymbol{L}^{\boldsymbol{m}}\right)^{\mathrm{T}}\right] \text {. }
$$

However, we caution readers that this modeled strain rate will not correspond to strain rates measured in situ, because we are solving for fabric evolution, not ice flow. The deformation of ice is not only a function of fabric (as modeled here) but of many co-dependent processes, which accommodate ice deformation (Thorsteinsson and others, 1999). Further, using the uniform-stress assumption results in ice that is too stiff (Montagnat and others, 2014), meaning the fabric evolves too much for the modeled strain rates compared with measured strain rates. Using NNIs partially alleviates this problem by allowing hard grains to deform.
Nevertheless, the cumulative modeled bulk strain, provides a good measure of the overall fabric evolution (in the context of the model), and is useful when comparing different model runs, as we do below. In order to compare results from our model with measured fabrics, the flow law constant, $\beta$ (Eqn (4)), and parameters controlling the local softness, $[\zeta, \xi]$ (Eqn (6)), need to be tuned to reproduce the observed fabric evolution in time. How much the strain rate is under-represented in the model can then be determined. The effects of changing these parameters in our model are discussed below.

\subsection{Recrystallization processes}

Once the velocity gradient and strain rates are calculated for each grain, the parameterizations of normal grain growth, polygonization and migration recrystallization (SIBM-N) are applied to the grain distribution before the grains are rotated into new orientations.

\subsubsection{Normal grain growth}

Under the tripartite paradigm, normal grain growth occurs when grain boundaries migrate, in order to reduce the overall grain-boundary energy. The grain growth is a function of boundary curvature, intrinsic properties (e.g. temperature, thickness, diffusivity of water molecules) and extrinsic material (e.g. impurities, bubbles). This grain growth can be described by a parabolic growth law (Alley and others, 1986), where the grain diameter, $D$, increases with time:

$$
D^{2}=K t+D_{0}^{2}
$$

where $K$ is the grain-growth factor, $t$ is time, $t_{0}$ is the initial time and $D_{0}$ is the grain diameter at time $t_{0}$. The graingrowth factor is a function of the intrinsic properties of the ice and the temperature:

$$
K=K_{0} \exp \left(-\frac{Q_{b}^{ \pm}}{\mathcal{R} T}\right)
$$

where $K_{0}$ is a constant that depends on the intrinsic properties of the grain boundaries, $Q_{\mathrm{b}}^{ \pm}$is the activation energy for 
grain-boundary self-diffusion, $\mathcal{R}$ is the gas constant and $T$ is the temperature. $Q_{\mathrm{b}}^{ \pm}$is $\sim 0.7 \times Q_{v}^{ \pm}$(Cuffey and Paterson, 2010 , p. 40) and, similarly to the activation energy for volume self-diffusion, $Q_{\mathrm{b}}^{ \pm}=Q_{\mathrm{b}}^{+}=81 \mathrm{~kJ} \mathrm{~mol}^{-1}$ for $T \geq T_{\text {太 }}$ and $Q_{\mathrm{b}}^{ \pm}=Q_{\mathrm{b}}^{-}=42 \mathrm{~kJ} \mathrm{~mol}^{-1}$ for $T<T_{\star}$ (Table 1 ). Extrinsic materials, such as dust particles, reduce the rate of boundary migration and can be described by a drag force on the boundary (Alley and others, 1986). This drag effectively reduces the grain-growth factor, $K$. We list the values used for these constants in Table 1.

Normal grain growth is then implemented by growing the diameter of the grain, $D$, according to Eqn (10). We reset the growth law after each recrystallization, such that $t_{0}$ and $D_{0}$ are the time and size immediately after the recrystallization.

\subsubsection{Polygonization}

Under the tripartite paradigm, a stable grain size is typically reached, even though grains continue growing through time and depth, because polygonization counteracts normal grain growth. Polygonization creates new grain boundaries within large ice grains, effectively dividing the grain in two. Large grains can become highly strained and experience differential stress, which is relieved by the organization of dislocations into subgrain boundaries (Alley, 1992). De La Chapelle and others (1998) determined that the minimum dislocation density needed to form a subgrain boundary is, $\rho_{\mathrm{p}}=5.4 \times 10^{10} \mathrm{~m}^{-2}$.

Because polygonization depends on reaching a minimum dislocation density, the rate of polygonization can be indirectly described through the dislocation density's rate of change. The dislocation density changes due to two dominant processes: it increases due to work hardening and decreases due to the absorption of dislocations by the grain boundary (Miguel and others, 2001). Therefore, the change in a grain's dislocation density can be described as

$$
\frac{\mathrm{d} \rho}{\mathrm{d} t}=\frac{\|\dot{\boldsymbol{\epsilon}}\|}{b D}-\alpha \rho \frac{K}{D^{2}},
$$

where the first term on the right describes work hardening: $\|\dot{\boldsymbol{\epsilon}}\|$ is the second invariant of the strain-rate tensor, $b$ is the length of the Burgers vectors and $D$ is the grain diameter (Montagnat and Duval, 2000). The second term describes the absorption of dislocations by the grain boundaries, $\alpha$ is an adjustable constant and $K$ is the grain-growth factor.

We implement polygonization such that once the minimum dislocation density is reached, a grain experiencing a differential stress may polygonize. Because our NNIs only modify the RSS and cannot directly apply a differential stress, we use a proxy for differential stress on a grain (Thorsteinsson, 2002). Grains that have a small amount of the applied stress resolved onto the basal plane (RSS) will likely be experiencing a differential stress from their neighboring grains which are deforming. Specifically, if the ratio of the magnitude of the RSS, $\mathcal{T}$, to the second invariant of the applied stress, $\left\|\boldsymbol{\sigma}^{\prime}\right\|$, is less than a given value, $\mathcal{T} /\left\|\boldsymbol{\sigma}^{\prime}\right\|<\delta$, and the dislocation density, $\rho$, in the grain is sufficient to form a subgrain wall $\left(\rho>\rho_{\mathrm{p}}\right)$, then the grain will polygonize. When a grain polygonizes, the orientation is changed by an angle, $\Delta \theta$, in a direction that increases the RSS, the grain size is halved and the dislocation density is reduced by $\rho_{\mathrm{p}}$. Values for these parameters are listed in Table 1. Polygonization tends to slow the development of fabric because grains that are oriented very close to the preferred orientation (small RSS) of the fabric will polygonize preferentially by the selection criteria. Because polygonization rotates the grains away from the preferred orientation, this process tends to weaken the fabric.

\subsubsection{Migration recrystallization (SIBM-N)}

According to the tripartite paradigm, through most of the depth of an ice sheet, the rate of fabric evolution is controlled by a balance between the grain growth, polygonization and grain rotation processes. However, migration recrystallization dominates fabric evolution at high temperatures (typically $>-10^{\circ} \mathrm{C}$; Duval and Castelnau, 1995). Migration recrystallization occurs when the stored strain energy (due to dislocations) in a grain is greater than the grain-boundary energy of a new strain-free grain. This new strain-free grain rapidly grows at the expense of the old grain (Duval and Castelnau, 1995). The stored energy due to dislocations, $E_{\mathrm{d}}$, can be estimated as

$$
E_{\mathrm{d}} \simeq \mu \rho G b^{2} \ln \left(\frac{R_{\mathrm{e}}}{b}\right),
$$

where $\mu$ is a constant, $G$ is the shear modulus and $R_{\mathrm{e}}$ is the mean average of the dislocation strain field range (Thorsteinsson, 2002). The energy associated with grain boundaries, $E_{\mathrm{c}}$, is

$$
E_{\mathrm{c}}=\frac{3 \gamma_{\mathrm{g}}}{D},
$$

where $\gamma_{\mathrm{g}}$ is the energy per unit area on the boundary (for high-angle boundaries). When $E_{\mathrm{d}}>E_{\mathrm{c}}$ it is energetically favorable to nucleate a new grain, which quickly grows to a diameter that scales with the effective stress, due to a balance between nucleation of grains and grain-boundary migration (e.g. Shimizu, 2008). The nucleated grain that grows most rapidly will be the one in the most energetically favorable position: about halfway between the compressional and tensional axes, which maximizes the resolved shear stress on the basal planes, causing them to deform easily (Alley, 1992). For uniaxial compression or pure shear, for example, this is $45^{\circ}$ from the axis of compression, while for simple shear, it is $45^{\circ}$ from the principal stress axis (Fig. 3). The new grain is initially strain-free and has a much lower strain energy than the surrounding grains, allowing it to grow. As the new grain grows preferentially at an orientation favorable for the bulk deformation (highest RSS), the fabric can change significantly when there are large numbers of migration recrystallization events.

We implement migration recrystallization by immediately creating a new grain when the dislocation energy, $E_{\mathrm{d}}$, exceeds the boundary energy, $E_{\mathrm{c}}$ (Eqns (13) and (14)). An old grain is replaced with a new 'strain-free' grain that has a dislocation density $\rho_{0}$ and a diameter that scales with the effective stress, $D \sim\left(\sigma_{k l}^{\prime} \sigma_{k l}^{\prime} / 2\right)^{-2 / 3}$ (Thorsteinsson, 2002; Shimizu, 2008). We assume the grain grows fast enough to reach a diameter of $D$ within a single time step. The new grain is given the orientation with the highest RSS (or softest orientation; Fig. 3), taken from a random distribution of 50 orientations in the applied stress state (Thorsteinsson, 2002).

\subsubsection{Lattice rotation}

If the surrounding ice is fixed, each grain rotates as it deforms, according to the standard continuum mechanics rotation rate tensor:

$$
\dot{\boldsymbol{\Omega}}^{\mathrm{p}}=\frac{1}{2}\left[\boldsymbol{L}^{\mathrm{C}}-\left(\boldsymbol{L}^{\mathrm{C}}\right)^{\mathrm{T}}\right],
$$




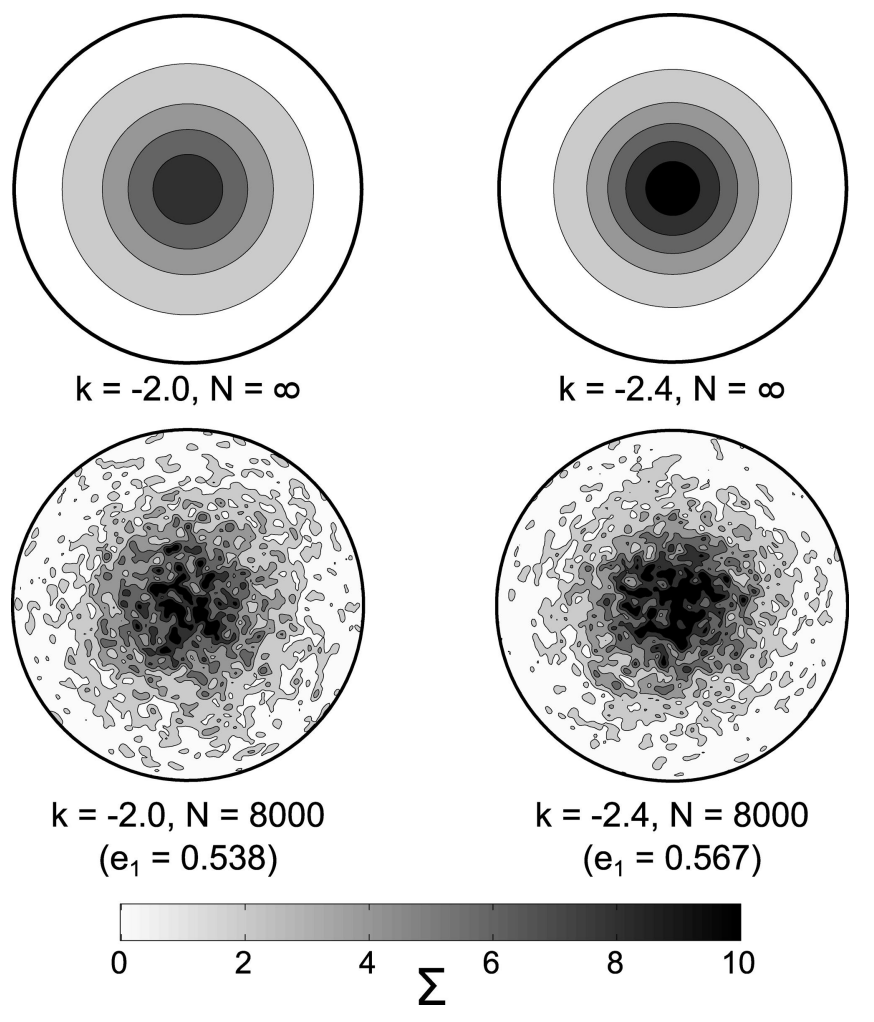

Fig. 4. Contoured Schmidt plot of the initial fabrics for both the constant-stress and Taylor Dome experiments. The fabrics are contoured at levels of $0,2 \Sigma, \ldots, 10 \Sigma . \Sigma$ is the standard deviation of the density of grains from the expected density for isotropic ice (Kamb, 1959). The upper two are contour plots of the continuous Watson distribution (an infinite number of grains), with concentration parameters $k=-2.0$ (left) and $k=-2.4$ (right). Two random 8000-grain fabrics generated from the upper Watson distributions are depicted in the lower two plots. The fabric generated from the $k=-2.0$ and $k=-2.4$ distributions have eigenvalues of $e_{1}=0.538$ and $e_{1}=0.567$, respectively.

where $\dot{\boldsymbol{\Omega}}^{\mathrm{p}}$ is the rotation rate of a single grain and $\boldsymbol{L}^{\mathrm{c}}$ is the velocity gradient of a grain in response to stress from Eqn (4). If the surrounding ice is rotating within the frame of reference, however, the model calculates a relative grain rotation rate:

$$
\dot{\Omega}^{*}=\dot{\Omega}^{b}-\dot{\Omega}^{p},
$$

where $\dot{\Omega}^{b}$ is the bulk rotation rate of the modeled ice in response to stress. The new orientation of the grain is then

$$
\vec{C}^{\prime}=\left(\boldsymbol{I}+t \dot{\boldsymbol{\Omega}}^{\star}\right) \vec{C} .
$$

\section{EXPERIMENTAL SET-UP}

The model domain is a 24000 -grain cuboid that is 20 grains wide, 20 grains deep and 60 grains high (Fig. 1). The cuboid is split into three vertically layered cubes of 8000 grains each and the middle layer is initialized with a different fabric to the top and bottom layers. Each layer of the initial fabric is generated using a Watson distribution (Kennedy and others, 2013). The top and bottom layers have a concentration parameter for the Watson distribution of $k_{\mathrm{tb}}=-2.0$. This results in a vertical single-maximum fabric, where the largest eigenvalue of the second-order orientation tensor is $e_{1}^{t b}=0.538$. The middle layer has a stronger fabric, with a concentration parameter of $k_{\mathrm{m}}=-2.4\left(e_{1}^{\mathrm{m}}=0.567\right)$. These concentration parameters are characteristic of ice fabrics found near the firn-ice transition in polar ice sheets, and correspond to fabrics at $\sim 100 \mathrm{~m}$ depth in Taylor Dome, East Antarctica (Kennedy and others, 2013), and at $<500 \mathrm{~m}$ depth at Dome C, East Antarctica (Wang and others, 2003; Durand and others, 2009). We measure the fabric variation through time by calculating the difference in the fabric $e_{1}$ eigenvalues between the middle and top (or bottom) layers: $\Delta e_{1}=e_{1}^{m}-e_{1}^{t}$. $e_{1}$ will best represent single-maximum-type grain distributions typically found in ice sheets. Initially, $\Delta e_{1}=0.029$. A contoured Schmidt plot of the initial fabrics is shown in Figure 4.

Over time, as the cuboid is stressed, $\Delta e_{1}$ will change and may become smaller than the uncertainty in eigenvalues, due to under-sampling the distribution with a finite number of grains. Durand and others (2006b) found the maximum under-sampling error to be $\delta=0.004$ for a distribution with 8000 grains. For this study, we consider the minimum separation resolvable above the error in the eigenvalue calculation to be twice the maximum error: $2 \delta \approx 0.01$. The fabric variation is then preserved, as long as $\Delta e_{1}>0.01$. In situ, the minimum separation required to measure a fabric anomaly will depend on the measurement technique, the number of grains sampled and the assumed in situ distribution of orientations.

To model how our fabrics respond to stress, we apply a constant temperature, $T$, and constant deviatoric stress, $\boldsymbol{\sigma}^{\prime}$, at each time step. The basic stress states within an ice sheet are uniaxial compression, pure shear and simple shear. The deviatoric-stress tensor for uniaxial compression has the form

$$
\boldsymbol{\sigma}_{\mathrm{u}}^{\prime}=\left(\begin{array}{ccc}
\frac{1}{2} \sigma_{\mathrm{u}}^{\prime} & 0 & 0 \\
0 & \frac{1}{2} \sigma_{\mathrm{u}}^{\prime} & 0 \\
0 & 0 & -\sigma_{\mathrm{u}}^{\prime}
\end{array}\right),
$$

the deviatoric-stress tensor for pure shear has the form

$$
\boldsymbol{\sigma}_{\mathrm{p}}^{\prime}=\left(\begin{array}{ccc}
\sigma_{\mathrm{p}}^{\prime} & 0 & 0 \\
0 & 0 & 0 \\
0 & 0 & -\sigma_{\mathrm{p}}^{\prime}
\end{array}\right)
$$

and the deviatoric-stress tensor for simple shear has the form

$$
\boldsymbol{\sigma}_{\mathrm{s}}^{\prime}=\left(\begin{array}{ccc}
0 & 0 & \sigma_{\mathrm{s}}^{\prime} \\
0 & 0 & 0 \\
\sigma_{\mathrm{s}}^{\prime} & 0 & 0
\end{array}\right) .
$$

We use $\sigma_{u, p, s}^{\prime}$ of 0.01 and $0.04 \mathrm{MPa}$ to provide a lower and upper bound on the characteristic deviatoric stresses typically found in ice sheets (Pettit and Waddington, 2003). The model calculates a strain rate at each time step, $\dot{\epsilon}_{i}^{\mathrm{m}}$, and then the fabric is evolved for the amount of time required to achieve a strain step of $0.001, t_{i}$. The total bulk strain undergone by the modeled ice is then $\epsilon=\sum \dot{\epsilon}_{i}^{\mathrm{m}} t_{i}$.

Ice in the vicinity of an ice divide will typically experience a regime of compressive stress before experiencing significant shear stress and, for simple ice-sheet geometries, simple-shear stresses are most important in the bottom half of the ice sheet (Cuffey and Paterson, 2010). Therefore we apply a constant compressive-stress regime (R1) to the modeled ice up to a total bulk strain of $\epsilon_{\mathrm{R} 1}$. We then apply a constant-shear-stress regime (R2) to the modeled ice up to a total bulk strain of $\epsilon_{R 2}$. The shearstress regimes consist of simple shear alone, a combination of simple shear and pure shear, or a combination of simple shear and uniaxial compression (Table 2). 
Table 2. Stress regimes, stress magnitude, NNI parameters and temperature values used in the constant-stress experiments. Two hundred and eighty-eight model runs were computed, where each run used a permutation of the listed values. $\sigma_{u}^{\prime}$ indicates uniaxial compression, $\sigma_{\mathrm{p}}^{\prime}$ indicates pure shear and $\sigma_{\mathrm{s}}^{\prime}$ indicates simple shear (Eqns (18-20))

\begin{tabular}{lcccc}
\hline $\mathrm{R} 1 \rightarrow \mathrm{R} 2$ & $\begin{array}{c}\sigma_{\mathrm{u}, \mathrm{p}}^{\prime} \\
\mathrm{MPa}\end{array}$ & $\begin{array}{c}\sigma_{\mathrm{s}}^{\prime} \\
\mathrm{MPa}\end{array}$ & $\mathrm{NNI}[\zeta, \xi]$ & $\mathrm{T}$ \\
& & & \\
\hline${ }^{\circ} \mathrm{C}$
\end{tabular}

Kennedy and others (2013) showed that the separation in eigenvalues between the fabric layers drops below 0.01 after 0.30 bulk strain in our model when undergoing uniaxial compression and pure shear. Simple shear, however, causes the fabric to rotate into a 'soft' orientation rather than a 'hard' orientation. Because the rate of grain rotation depends on the velocity gradient, the stronger fabric (soft in simple shear) will evolve more quickly than the weaker fabric (hard in simple shear). Therefore, the fabric separation between layers can increase in simple shear as the fabric evolves with each time step. This will cause fabrics to maintain their separation to a higher modeled bulk strain than 0.30 . However, after 0.35 strain in our model runs dominated by high magnitudes of uniaxial compression or pure shear, the time step necessary for a 0.001 strain-step increases by over three orders of magnitude due to stress hardening. Further evolution becomes computationally impractical, because time steps that result in realistic amounts of recrystallization become too small. We therefore set $\epsilon_{\mathrm{R} 2}=0.35$ in our model, to capture the possible enhancement of the eigenvalue separation between fabric layers due to simple shear, but do not evolve the fabric further to avoid excessively large time steps. Kennedy and others (2013) found that for dome-like and ridge-like ice sheets, 0.30 modeled bulk strain corresponds to evolution through $\sim 200 \mathrm{ka}$ while $\sim 0.20$ bulk strain was $\sim 100 \mathrm{ka}$. We then set $\epsilon_{\mathrm{R} 1}=0.20$ so that $\epsilon_{\mathrm{R} 1}$ corresponds to about half of our fabric evolution time.

\section{RESULTS AND DISCUSSION}

We evolve the layered fabric shown in Figure 4 through each of the 288 permutations of the stress style, stress magnitudes, NNI parameters and temperature values shown in Table 2. Figure 5 shows a contoured ternary plot of the eigenvalues for every time step of all 288 model runs. The fabrics all evolve towards the expected single-maximumtype fabrics found in ice sheets. The layered fabric initially has an eigenvalue separation of $\Delta e_{1}=0.029$ between the top/bottom layer and the middle layer. The total amount of strain where $\Delta e_{1}$ is greater than the under-sampling error (0.01) depends on the rates and magnitudes of several competing processes: grain rotation; NNIs; the stress regime; and the resulting amount of recrystallization.

We focus on the results that best illustrate the effects of simple shear, temperature and NNIs on the eigenvalue separation. Because each of these processes affects the rate

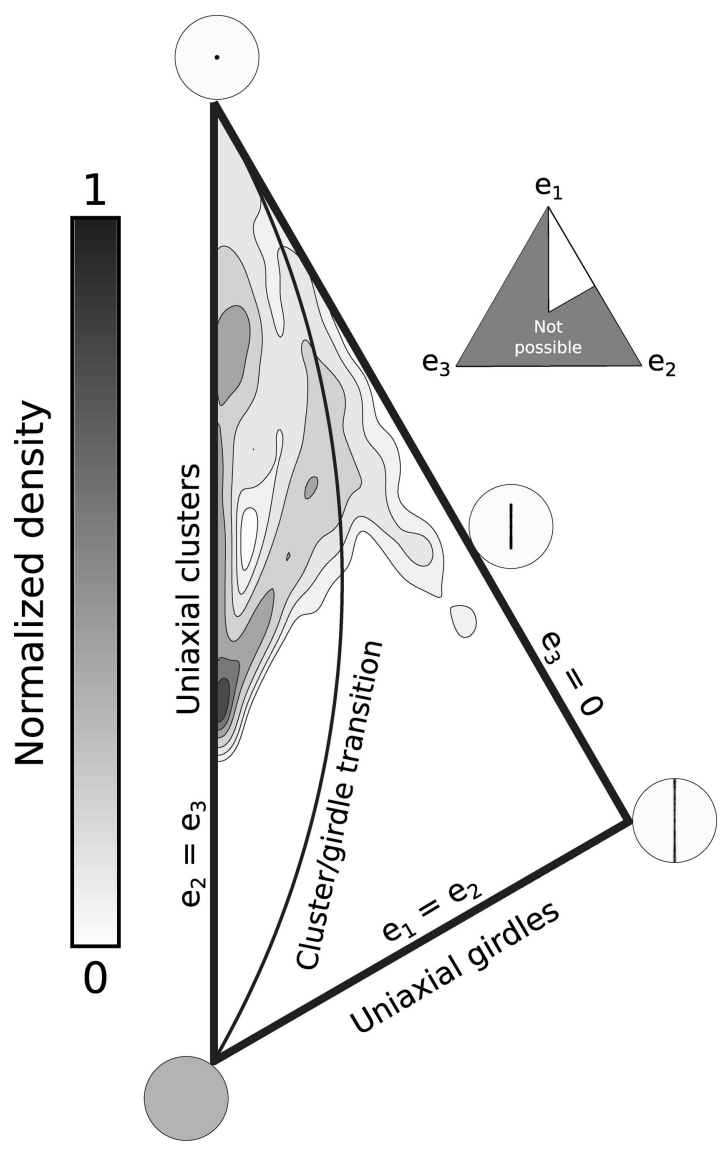

Fig. 5. Contoured ternary plot of the eigenvalues of the fabrics for every time step of all 288 model runs. Because by definition $e_{1}>e_{2}>e_{3}$, only one-sixth of the equilateral triangle is used. The fabric density, $\rho_{\mathrm{f}}$, has been normalized by the maximum fabric density $\left(\rho_{\mathrm{f}} / \max \left(\rho_{\mathrm{f}}\right)\right)$. Example Schmidt plots show the fabrics with the eigenvalues directly adjacent to them.

and type of recrystallization events, recrystallization is discussed throughout.

\subsection{Simple shear}

Figure 6 shows the evolution of the fabric for the stress regimes $(\mathrm{R} 1 \rightarrow \mathrm{R} 2)$ of: uniaxial compression only $\left(\boldsymbol{\sigma}_{\mathrm{u}}^{\prime} \rightarrow \boldsymbol{\sigma}_{\mathrm{u}}^{\prime}\right)$; uniaxial compression to simple shear $\left(\boldsymbol{\sigma}_{\mathrm{u}}^{\prime} \rightarrow \boldsymbol{\sigma}_{\mathrm{s}}^{\prime}\right)$; and uniaxial compression to uniaxial compression plus simple shear $\left(\boldsymbol{\sigma}_{\mathrm{u}}^{\prime} \rightarrow \boldsymbol{\sigma}_{\mathrm{u}}^{\prime}+\boldsymbol{\sigma}_{\mathrm{s}}^{\prime}\right)$. The fabrics were evolved at $-30^{\circ} \mathrm{C}$ with mild $\mathrm{NNI}([\zeta, \xi]=[6,1]$; Eqn (6)) and low stress magnitudes $\left(\sigma_{\mathrm{u}, \mathrm{s}}^{\prime}=0.01 \mathrm{MPa}\right)$.

In the $\sigma_{\mathrm{u}}^{\prime} \rightarrow \sigma_{\mathrm{s}}^{\prime}$ model run, the eigenvalue separation, $\Delta e_{1}$, remains above the under-sampling error for the entire experiment. When compared with the $\boldsymbol{\sigma}_{u}^{\prime} \rightarrow \boldsymbol{\sigma}_{u}^{\prime}$ run, fabric evolution is slowed, polygonization events happen less frequently and there is some migration recrystallization (SIBM-N) happening at high modeled bulk strains $(>0.30)$. The fabric evolution is slowed because at 0.20 modeled bulk strain, the fabrics are already mostly concentrated near vertical and the grains at the periphery of the distribution are now in the hardest orientation in simple shear (Fig. 3). These peripheral grains rotate towards the vertical slowly, causing $e_{1}$ to increase slowly. Likewise, the polygonization frequency is reduced because the grains in a hard orientation in simple shear (and therefore likely to be experiencing a bending moment; $\mathcal{T} /\left\|\boldsymbol{\sigma}^{\prime}\right\|<\delta$, see Section 3.2.2) have not yet undergone enough modeled deformation to have a high 

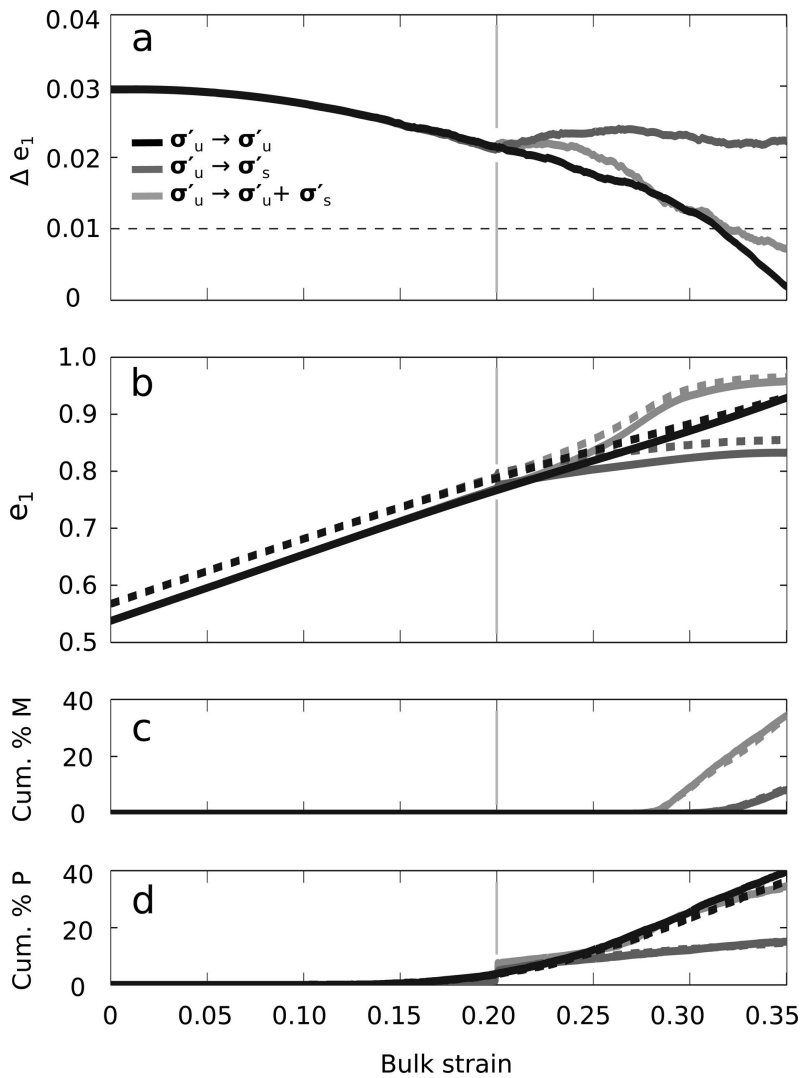

Fig. 6. The effects of simple shear on the evolution of the layered fabric (Fig. 4). The fabric was evolved at $-30^{\circ} \mathrm{C}$ with mild NNI $\left([\zeta, \xi]=[6,1]\right.$; Eqn (6)) and low stress magnitudes $\left(\sigma_{\mathrm{u}, \mathrm{s}}^{\prime}=0.01 \mathrm{MPa}\right)$ for the stress regimes $(\mathrm{R} 1 \rightarrow \mathrm{R} 2)$ of: uniaxial compression only $\left(\boldsymbol{\sigma}_{u}^{\prime} \rightarrow \boldsymbol{\sigma}_{u}^{\prime}\right.$, black curves); uniaxial compression to simple shear $\left(\sigma_{u}^{\prime} \rightarrow \sigma_{s}^{\prime}\right.$, dark gray curves) and uniaxial compression to uniaxial compression plus simple shear $\left(\boldsymbol{\sigma}_{\mathrm{u}}^{\prime} \rightarrow \boldsymbol{\sigma}_{\mathrm{u}}^{\prime}+\boldsymbol{\sigma}_{\mathrm{s}}^{\prime}\right.$, light gray curves). (a) The $\Delta e_{1}$ eigenvalue separation between the top/bottom and middle fabric layers. The horizontal dashed curve indicates the under-sampling error threshold, where $\Delta e_{1}$ may not be resolvable. (b-d) The fabric evolution (b), the cumulative percent of grains that have undergone migration recrystallization (c) and the cumulative percent of grains that have undergone a polygonization event (d). Solid curves indicate the top/bottom layer, while thick dashed curves indicate the middle layer. In all plots, light gray vertical lines mark the change from R1 to R2.

dislocation density. The grains that have a high dislocation density are very close to vertical and located in a soft orientation (therefore unlikely to be experiencing a bending moment). Further, migration recrystallization events happen at the high modeled bulk strains for this run because the soft grains that already have high dislocation density increase their dislocation density further, to the point where migration recrystallization is possible even at such low temperatures. This agrees with observations by Kipfstuhl and others (2009), which showed that recrystallization can be active at much lower temperatures than previously suggested. Because grains that undergo migration recrystallization are given a random orientation with a high RSS, they are likely to end up with an orientation either close to vertical or close to the direction of shearing (Fig. 3). The grains that end up pointing close to vertical will not strongly affect the fabric eigenvalues, as they stay within the vertically clustered distribution. The fabric in the $\boldsymbol{\sigma}_{\mathrm{u}}^{\prime} \rightarrow \boldsymbol{\sigma}_{\mathrm{s}}^{\prime}$ model run remains largely unaffected by the small number of migration recrystallization events.
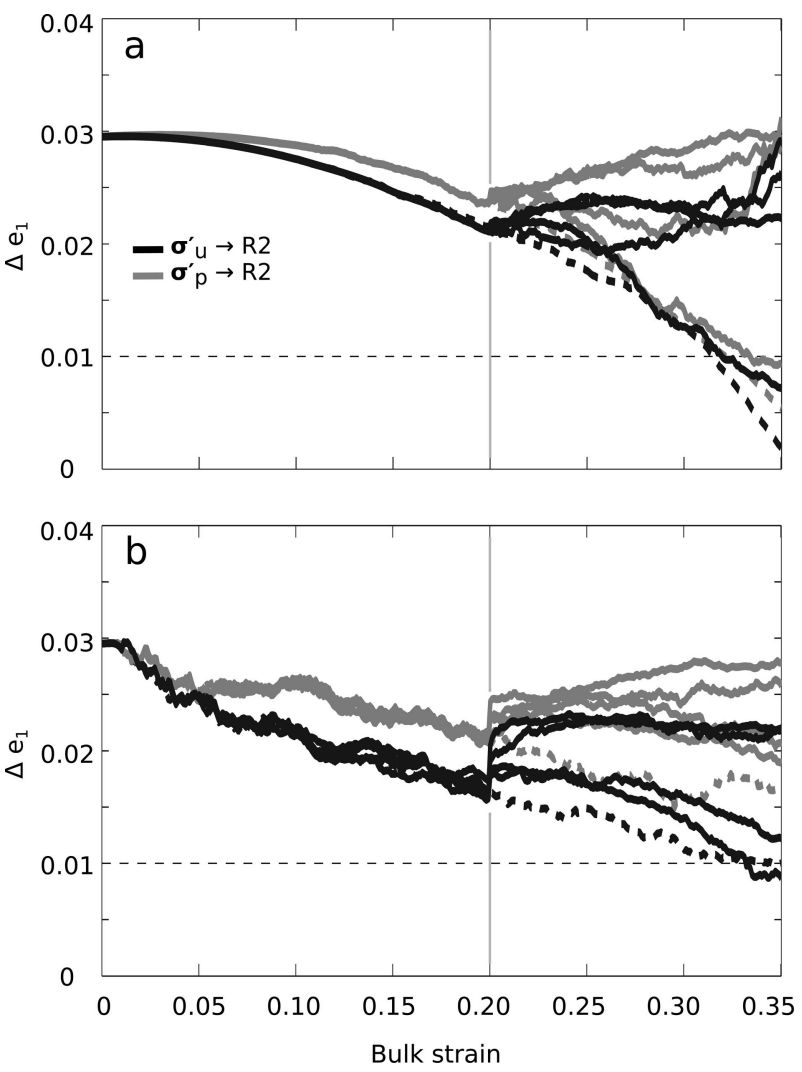

Fig. 7. The effects of simple shear on the evolution of the layered fabric (Fig. 4) eigenvalue separation between the top/bottom and middle fabric layers. The fabric was evolved at $-30^{\circ} \mathrm{C}$ with mild NNI $([\zeta, \xi]=[6,1]$; Eqn (6)) in every permutation of the stress regimes $(\mathrm{R} 1 \rightarrow \mathrm{R} 2)$ and stress magnitudes $\left(\sigma_{\mathrm{u}, \mathrm{p}, \mathrm{s}}^{\prime}\right)$ shown in Table 2. Black curves indicate runs that started with uniaxial compression, $\sigma_{u^{\prime}}^{\prime}$ while gray curves indicate runs that started with pure shear, $\boldsymbol{\sigma}_{\mathrm{p}}^{\prime}$. (a) Runs with a low stress magnitude initially $\left(\sigma_{u, p}^{\prime}=0.01 \mathrm{MPa}\right)$. (b) Runs with a high stress magnitude initially $\left(\sigma_{u, p}^{\prime}=0.04 \mathrm{MPa}\right)$. In both plots, the horizontal dashed line (b) indicates the undersampling error threshold, where $\Delta e_{1}$ may not be resolvable, and light gray vertical lines in both plots mark the change from R1 to R2.

In the $\boldsymbol{\sigma}_{\mathrm{u}}^{\prime} \rightarrow \boldsymbol{\sigma}_{\mathrm{u}}^{\prime}+\boldsymbol{\sigma}_{\mathrm{s}}^{\prime}$ model run, $\Delta \mathrm{e}_{1}$ remains above the under-sampling error to just slightly higher modeled bulk strains than in the $\sigma_{\mathrm{u}}^{\prime} \rightarrow \boldsymbol{\sigma}_{\mathrm{u}}^{\prime}$ run. Simple shear does not slow the fabric evolution in this case because the majority of grains will have a large RSS (Fig. 3), causing a rapid evolution of the fabric to the point where the fabric is too strongly orientated to maintain much separation. Both polygonization and migration recrystallization are active in this model run, due to the high RSS, which causes high modeled strain rates and a rapid build-up of dislocations. In this stress state, migration recrystallization will grow grains in almost any orientation. However, these grains will rapidly rotate to a vertical orientation and the rate of migration recrystallization events seen here does not change the fabric eigenvalues.

Figure 7 shows the $\Delta e_{1}$ evolution for all model runs at $-30^{\circ} \mathrm{C}$ with mild $\mathrm{NNI}$ (every permutation of stress and stress magnitude shown in Table 2). In all cases, simple shear stress causes the modeled bulk strain at which the eigenvalue separation stays above the under-sampling error $\left(\Delta e_{1}>0.01\right)$ to either remain the same (once) or increase (15 times). 

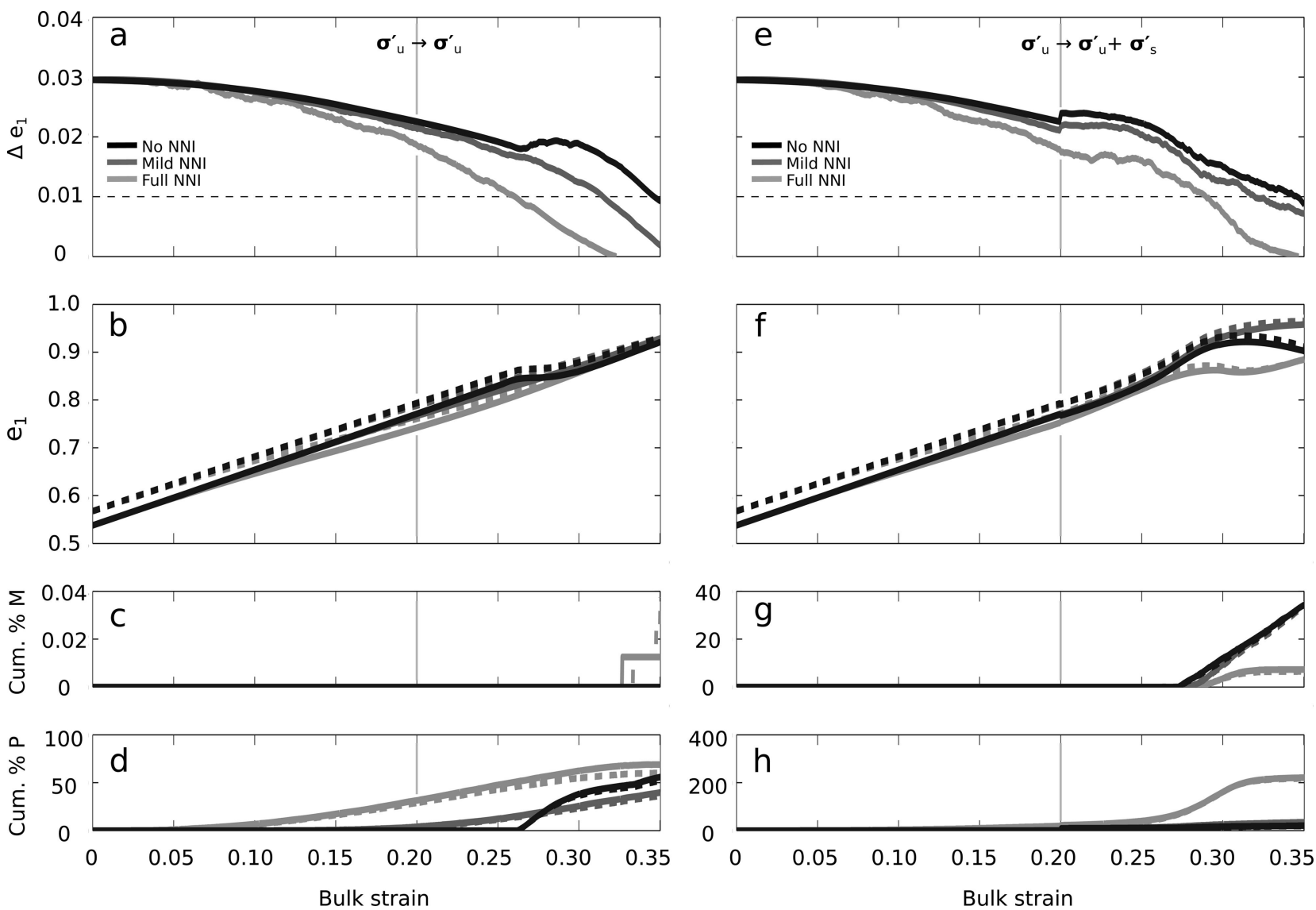

Fig. 8. The effects of NNI on the evolution of the layered fabric (Fig. 4). The fabric was evolved at $T=-30^{\circ} \mathrm{C}$ with each of the NNIs shown in Table 2. (a-d) The evolution of uniaxial compression only $\left(\boldsymbol{\sigma}_{u}^{\prime} \rightarrow \boldsymbol{\sigma}_{u}^{\prime}\right)$ with a low stress magnitude $\left(\sigma_{u}^{\prime}=0.01 \mathrm{MPa}\right)$. (e-h) The evolution of uniaxial compression plus simple shear $\left(\boldsymbol{\sigma}_{\mathrm{u}}^{\prime} \rightarrow \boldsymbol{\sigma}_{\mathrm{u}}^{\prime}+\boldsymbol{\sigma}_{\mathrm{s}}^{\prime}\right)$ with a low stress magnitude $\left(\sigma_{\mathrm{u}, \mathrm{s}}^{\prime}=0.1 \mathrm{MPa}\right)$. Black curves indicate no $\mathrm{NNI}$ $([\zeta, \xi]=[1,0]$; Eqn (6)), dark gray curves indicate mild NNI $([\zeta, \xi]=[6,1])$ and light gray curves indicate full NNI $([\zeta, \xi]=[1,1])$. (a) and (e) show the $\Delta e_{1}$ eigenvalue separation between the fabric's top/bottom and middle layers, and horizontal dashed lines indicate the undersampling error threshold, where $\Delta e_{1}$ may not be resolvable. $(b-d)$ and $(f-h)$ show the fabric evolution, the cumulative percent of grains that have undergone migration recrystallization and the cumulative percent of grains that have undergone a polygonization event, respectively. Solid curves indicate the fabric's top/bottom layer, and dashed curves indicate the middle layer. In all plots, the light gray vertical lines mark the change from R1 to R2.

\subsection{Nearest-neighbor interaction}

Figure 8 shows the repeated evolution of the same layered fabric with no NNI $([\zeta, \xi]=[1,0]$; Eqn (6)), mild NNI $([\zeta, \xi]=[6,1])$ and full NNI $([\zeta, \xi]=[1,1])$ in the two stress regimes of uniaxial compression only $\left(\sigma_{\mathrm{u}}^{\prime} \rightarrow \sigma_{\mathrm{u}}^{\prime}\right.$; Fig. 8a-d) and uniaxial compression to uniaxial compression plus simple shear $\left(\boldsymbol{\sigma}_{\mathrm{u}}^{\prime} \rightarrow \boldsymbol{\sigma}_{\mathrm{u}}^{\prime}+\boldsymbol{\sigma}_{\mathrm{s}}^{\prime}\right.$; Fig. 8e-h). The fabric was evolved using $T=-30^{\circ} \mathrm{C}$ and low stress magnitudes $\left(\sigma_{\mathrm{u}, \mathrm{s}}^{\prime}=0.01 \mathrm{MPa}\right)$ for both the stress regimes. Higher amounts of NNIs reduce the fabric separation at earlier modeled bulk strains. This happens because NNIs minimize the modeled strain-rate differences between neighboring grains, such that the grains tend to evolve towards vertical more slowly. This causes the grains to spend more time in a higher strain-rate orientation, which increases the dislocation density. A higher dislocation density allows the recrystallization processes to happen at an earlier modeled bulk strain. Polygonization then slows the fabric evolution by moving grains away from vertical. This affects the stronger fabric preferentially, as it has more hard grains that are prone to polygonization. Therefore, higher levels of NNls decrease the modeled bulk strain at which $\Delta e_{1}$ remains $>0.01$.

\subsection{Temperature}

Figure 9 shows the repeated evolution of the same layered fabric for the temperatures $T=-30,-15,-10,-5^{\circ} \mathrm{C}$ in the two stress regimes of uniaxial compression only $\left(\boldsymbol{\sigma}_{\mathrm{u}}^{\prime} \rightarrow \boldsymbol{\sigma}_{\mathrm{u}}^{\prime}\right.$; Fig. 9a-d) and uniaxial compression to uniaxial compression plus simple shear $\left(\boldsymbol{\sigma}_{\mathrm{u}}^{\prime} \rightarrow \boldsymbol{\sigma}_{\mathrm{u}}^{\prime}+\boldsymbol{\sigma}_{\mathrm{s}}^{\prime}\right.$; Fig. 9e-h). The fabric was evolved using mild NNI $([\zeta, \xi]=[6,1]$; Eqn (6)) and low stress magnitudes $\left(\sigma_{\mathrm{u}, \mathrm{s}}^{\prime}=0.01 \mathrm{MPa}\right)$ for both the stress regimes. The results from these runs fall into two sets: $T=-30,-15^{\circ} \mathrm{C}$ and $T=-10,-5^{\circ} \mathrm{C}$. The evolution of the fabric does not differ significantly within a set, but there is a large change in the fabric evolution between the sets, due to the step change in the activation energy, $Q_{b}$ (Eqn (11), Table 1). At any given modeled bulk strain, the change in activation energy results in a decrease in the eigenvalue separation, $\Delta e_{1}$, a slowdown of the $e_{1}$ evolution and an increase in the polygonization events. Temperature, therefore, does not change the fabric evolution in the modeled bulk strain, except when crossing the activation energy threshold.

Nevertheless, because ice at a higher temperature has a larger flow parameter $(\mathcal{A}(T)$, Eqn (4)), the ice will deform more quickly at higher temperatures. The actual time 

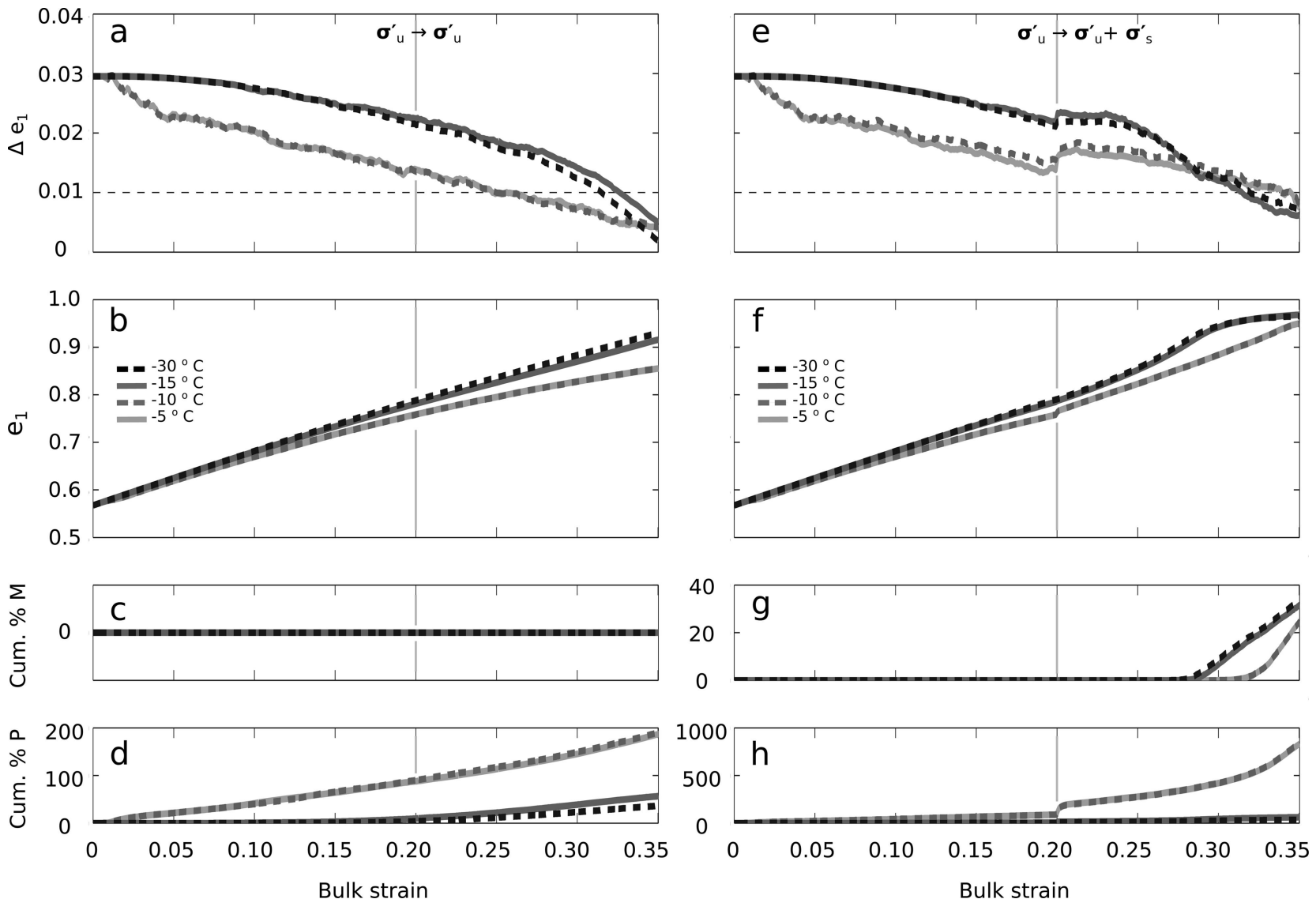

Fig. 9. The effects of temperature on the evolution of the layered fabric (Fig. 4). The fabric was evolved with mild NNI $([\zeta, \xi]=[6,1]$; Eqn $(6))$ in each of the temperature regimes shown in Table $2\left(T=-30,-15,-10\right.$ and $\left.-5^{\circ} \mathrm{C}\right)$. (a-d) The evolution of uniaxial compression only $\left(\boldsymbol{\sigma}_{\mathrm{u}}^{\prime} \rightarrow \boldsymbol{\sigma}_{\mathrm{u}}^{\prime}\right)$ with a low stress magnitude $\left(\sigma_{\mathrm{u}}^{\prime}=0.01 \mathrm{MPa}\right)$. (e-h) The evolution of uniaxial compression plus simple shear $\left(\boldsymbol{\sigma}_{\mathrm{u}}^{\prime} \rightarrow \boldsymbol{\sigma}_{\mathrm{u}}^{\prime}+\boldsymbol{\sigma}_{\mathrm{s}}^{\prime}\right)$ with a low stress magnitude $\left(\sigma_{\mathrm{u}, \mathrm{s}}^{\prime}=0.01 \mathrm{MPa}\right)$. The black dashed curves indicate temperatures of $T=-30^{\circ} \mathrm{C}$, the solid dark gray curves indicate $T=-15^{\circ} \mathrm{C}$, the dashed dark gray curves indicate $T=-10^{\circ} \mathrm{C}$ and the light gray curves indicate $T=-5^{\circ} \mathrm{C}$. (a) and (e) show the $\Delta \mathrm{e}_{1}$ eigenvalue separation between the top/bottom and middle fabric layers, and the horizontal dashed line indicates the under-sampling error threshold where $\Delta e_{1}$ may not be resolvable. $(\mathrm{b}-\mathrm{d})$ and $(\mathrm{f}-\mathrm{h})$ show the fabric evolution, the cumulative percent of grains that have undergone migration recrystallization and the cumulative percent of grains that have undergone a polygonization event, respectively. The light gray vertical lines in all plots mark the change from R1 to R2.

required for the modeled ice to reach any given bulk strain will then be shorter at higher temperatures. This also means that, for model runs that drop below an $e_{1}$ separation of 0.01 at the same modeled bulk strain, the actual time elapsed will be much shorter for runs at high temperatures (Fig. 10). Critically, migration recrystallization (SIBM-N) events are reduced for a given modeled bulk strain at the higher temperatures because of the very large number of polygonization events (which both depend on and reduce the dislocation density). Yet, because the time required to reach any given modeled bulk strain will be shorter, the earlier onset of migration recrystallization for higher temperatures still holds true.

\subsection{Further discussion}

In all of our experiments, a variation in fabric is either preserved or enhanced under shear stresses. There is a 'window of opportunity' in which the separation of eigenvalues is sufficient to observe the variation before the fabric becomes too strongly oriented to maintain much separation. The length of time this window is open depends on the magnitude of the initial fabric variation, the initial strength of the weaker fabric, the magnitude of the applied stress, the strength of the NNIs and the resultant number of

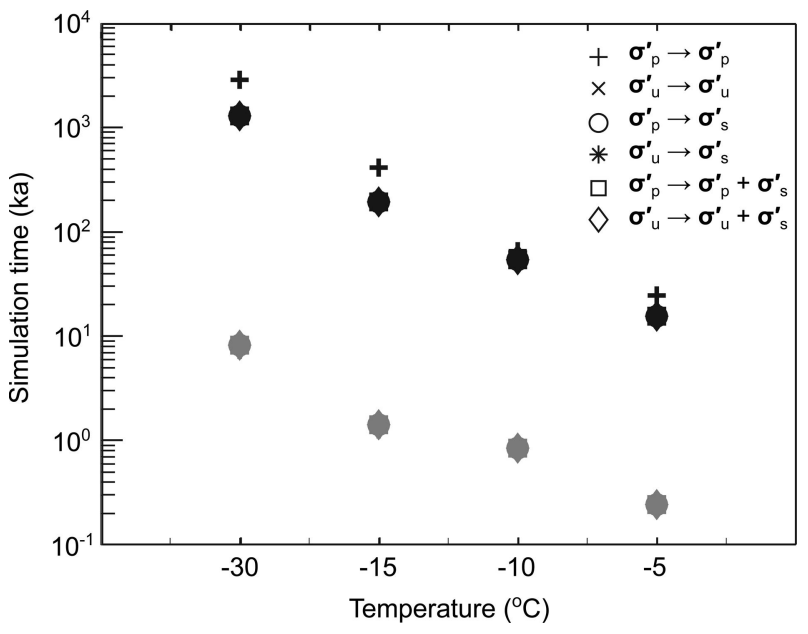

Fig. 10. The total simulation time to evolve the fabric to 0.35 bulk strain for our different model runs. Time is shown on a logarithmic scale. Black symbols indicate runs with low stress magnitudes $\left(\sigma_{\mathrm{u}, \mathrm{p}, \mathrm{s}}^{\prime}=0.01 \mathrm{MPa}\right)$ and gray symbols indicate runs with high stress magnitudes $\left(\sigma_{\mathrm{u}, \mathrm{p}, \mathrm{s}}^{\prime}=0.04 \mathrm{MPa}\right)$. 

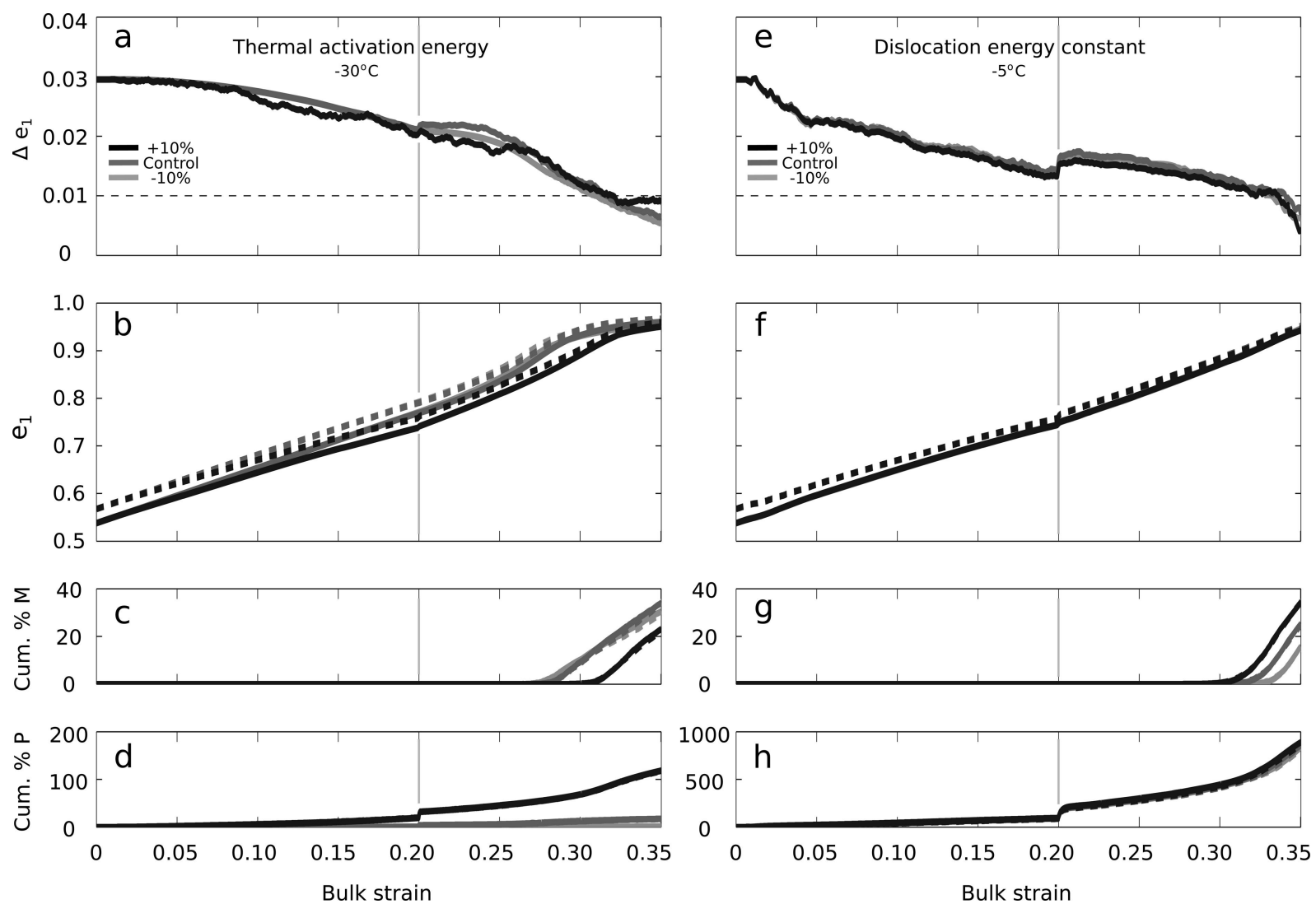

Fig. 11. Sensitivity to the model parameters (Table 1). (a-d) The result of varying the thermal activation energy, $Q$, at $T=-30^{\circ} \mathrm{C}$. (e-h) The result of varying the dislocation energy constant, $\mu$, at $T=-5^{\circ} \mathrm{C}$. Dark gray curves indicate the control run, while black curves indicate a $10 \%$ increase in the parameter values and light gray curves indicate a $10 \%$ decrease in the parameter values. (a) and (e) show the $\Delta \mathrm{e}_{1}$ eigenvalue separation between the fabric's top/bottom and middle layers, and horizontal dashed lines indicate the under-sampling error threshold where $\Delta e_{1}$ may not be resolvable. (b-d) and ( $\left.f-h\right)$ show the fabric evolution, the cumulative percent of grains that have undergone migration recrystallization and the cumulative percent of grains that have undergone a polygonization event respectively. Solid curves indicate the fabric's top/bottom layer, and dashed curves indicate the middle layer. In all plots, the light gray vertical lines mark the change from R1 to R2.

recrystallization events. If the initial fabric variation is sufficiently large, the weaker initial fabric controls the time the window is open - the window closes as the weaker fabric reaches the maximum fabric strength (the stronger fabric reaches the maximum fabric strength before the weaker fabric).

By finding the modeled strain (and therefore time) at which the $e_{1}$ separation is $<0.01$, we can determine how long the window stays open. Our model results suggest that the window will stay open at least through 0.3 modeled bulk strain in most of the modeled constant-stress regimes, and shear stress will keep the window open well past 0.35 modeled bulk strain. The total simulated time for any particular run is shown in Figure 10 and ranges from a few hundred years in the warmest, highest-stress cases to a few hundred thousand years in the coldest, lowest-stress cases (typical of an ice divide).

However, the parameter values in Table 1 may be different in situ, because we are not modeling a specific glacier. In order to test the sensitivity of our model to these parameters, we ran a variety of experiments varying parameters with an increase or decrease of $10 \%$. We tested changes in the isotropic ice softness by varying (1) $\beta$ (Eqn (4)); the grain growth (which influences the change in dislocation density and the rates of polygonization and SIBM-N) by varying (2) the intrinsic grain growth factor, $K_{0}$, and (3) the thermal activation energy, $Q$ (Eqns (11) and (12)); and the migration recrystallization (SIBM-N) threshold by varying (4) the dislocation energy constant, $\mu$ (Eqn (13)). These four parameters together allow us to vary all the processes captured in our model. For each parameter, we computed a set of model runs at $T=-30^{\circ} \mathrm{C}$, low uniaxial compression, low shear stress and mild NNIs. We computed another set of model runs at $T=-5^{\circ} \mathrm{C}$ with the same stresses and NNIs. Each set consists of a control run, a run with a $10 \%$ increase in the parameter and a run with a $10 \%$ decrease in the parameter. In total, we computed runs for four parameters, each in two temperature regimes, with three values for the parameters for 24 more model runs.

Most of the model runs are not presented here as they show only negligible effects on the fabric evolution (the changes are smaller than the width of the plot lines). The only significant changes in results we see occur when varying the thermal activation energy, $Q$, at $T=-30^{\circ} \mathrm{C}$ (Fig. 11a-d) and when varying the dislocation energy constant, $\mu$, at $T=-5^{\circ} \mathrm{C}$ (Fig. $11 \mathrm{e}-\mathrm{h}$ ). An increase in the thermal activation energy at $T=-30^{\circ} \mathrm{C}$ caused more polygonization events, fewer migration recrystallization events and a slower fabric evolution (Fig. 11b-d). However, because these changes are small, the $e_{1}$ separation is negligibly affected (Fig. 11a). Decreasing the activation energy had negligible effects.

Increasing the dislocation energy constant (Fig. 11g) causes a small increase in migration recrystallization events 
and, likewise, there was a small decrease in migration recrystallization events when the dislocation energy constant was decreased. Despite this change in migration recrystallization, the fabric evolution, polygonization and $e_{1}$ separation were negligibly affected (Fig. 11e, $f$ and $h$ ). Therefore, we conclude that our model is insensitive to small changes in parameter values and the conclusions presented above are robust.

It should also be noted that the fabric eigenvalues are not a complete description of the ice, and layers with the same eigenvalues may have different distributions of dislocation densities. It is possible for a fabric that has evolved to its maximum $e_{1}$ eigenvalue and/or lost the distinction between its layers $\left(\Delta e_{1}<0.01\right)$ to have its layers separate again due to different levels of recrystallization. We did not observe this re-separation in any of our model runs, but it may be seen with fabrics evolved to higher bulk strains than were modeled.

\section{CONCLUSIONS}

Our model predicts that for constant shear-stress regimes, modeled bulk strains $>0.35$ (for time, see Fig. 10) are necessary to rid glacial ice of its past 'memory' of fabrics and stress states. In our model, shear stress preserves a subtle variation in fabric longer than compressive-stress regimes and may act to enhance the fabric variation in certain stress regimes by rotating grains into softer orientations and reducing the number of polygonization events.

Our model further predicts that temperature does not affect the modeled bulk strain at which the fabric variation is sufficient to be observed, except when crossing a thermal activation energy threshold. The model shows that the much higher levels of recrystallization observed in warm, fastflowing ice are balanced by the increased modeled strain rates and grain rotation, such that the fabric variation may be observable past 0.35 modeled bulk strain. For any combination of the modeled stresses or temperatures, migration recrystallization (SIBM-N) does not rid the modeled fabric of its memory. However, using higher amounts of NNIs within the model reduces the fabric variation for a given modeled bulk strain.

We therefore conclude that fabric variation below the firn-ice transition, can be preserved in polar ice sheets. Because the microstructural processes active in the firn layer are dependent on climate variables, it is possible that the fabric variations arise from climate variations. In order to quantify the effects of climatic changes on the microstructure evolution in the firn layer, more work is needed. A microstructure model that is able to simulate multiple fabrics with a statistically relevant number of grains, that captures the dynamics in the firn and ice region, and is coupled to a flow model, is needed.

\section{ACKNOWLEDGEMENTS}

We thank the Scientific Editor Sérgio H. Faria, Paul Duval and an anonymous reviewer for detailed comments that considerably improved the manuscript. We also thank Martin Truffer, Ed Bueler and Christina Carr for help throughout the preparation of this manuscript. The work presented here was supported by US National Science Foundation (NSF) grants OPP\#0948247, OPP\#0940650 and OPP\#0636795.

\section{REFERENCES}

Adams EE and Miller DA (2003) Ice crystals grown from vapor onto an orientated substrate: application to snow depth-hoar development and gas inclusions in lake ice. J. Glaciol., 49(164), 8-12 (doi: 10.3189/172756503781830953)

Alley RB (1992) Flow-law hypotheses for ice-sheet modeling. J. Glaciol.. 38(129), 245-256

Alley RB, Perepezko JH and Bentley CR (1986) Grain growth in polar ice: I. Theory. J. Glaciol., 32(112), 415-424

Alley RB, Saltzman ES, Cuffey KM and Fitzpatrick JJ (1990) Summertime formation of depth hoar in central Greenland. Geophys. Res. Lett., 17(13), 2393-2396 (doi: 10.1029/ GL017i013p02393)

Alley RB, Gow AJ and Meese DA (1995) Mapping c-axis fabrics to study physical processes in ice. J. Glaciol., 41(137), 197-203

Arnaud L, Weiss J, Gay M and Duval P (2000) Shallow-ice microstructure at Dome Concordia, Antarctica. Ann. Glaciol., 30, 8-12 (doi: 10.3189/172756400781820813)

Benson CS (1962) Stratigraphic studies in the snow and firn of the Greenland ice sheet. SIPRE Res. Rep. 70

Budd WF and Jacka TH (1989) A review of ice rheology for ice sheet modelling, Cold Reg. Sci. Technol., 16(2), 107-144 (doi: 10.1016/0165-232X(89)90014-1)

Carns R, Waddington ED, Pettit EC and Warren SG (2010) A model of grain growth and crystal fabric in polar snow and firn. AGU Fall Meeting Abstracts, C33D-0572

Castelnau O, Duval P, Lebensohn RA and Canova GR (1996) Viscoplastic modeling of texture development in polycrystalline ice with a self-consistent approach: comparison with bound estimates. J. Geophys. Res., 101(B6), 13 851-13868 (doi: 10.1029/96JB00412)

Colbeck SC (1983) Theory of metamorphism of dry snow. J. Geophys. Res., 88(C9), 5475-5482 (doi: 10.1029/ JC088iC09p05475)

Cuffey KM and Paterson WSB (2010) The physics of glaciers, 4th edn. Butterworth-Heinemann, Oxford

De La Chapelle S, Castelnau O, Lipenkov V and Duval P (1998) Dynamic recrystallization and texture development in ice as revealed by the study of deep ice cores in Antarctica and Greenland. J. Geophys. Res., 103(B3), 5091-5105 (doi: 10.1029/97JB02621)

DiPrinzio CL, Wilen LA, Alley RB, Fitzpatrick JJ, Spencer MK and Gow AJ (2005) Fabric and texture at Siple Dome, Antarctica. J. Glaciol., 51(173), 281-290 (doi: 10.3189/ 172756505781829359)

Durand G, Gagliardini O, Thorsteinsson T, Svensson A, Kipfstuhl S and Dahl-Jensen D (2006a) Ice microstructure and fabric: an upto-date approach for measuring textures. J. Glaciol., 52(179), 619-630 (doi: 10.3189/172756506781828377)

Durand G and 10 others (2006b) Effect of impurities on grain growth in cold ice sheets. J. Geophys. Res., 111(F1), F01015 (doi: 10.1029/2005JF000320)

Durand G and 8 others (2007) Change in ice rheology during climate variations: implications for ice flow modelling and dating of the EPICA Dome C core. Climate Past, 3(1), 155-167 (doi: 10.5194/cp-3-155-2007)

Durand $G$ and 7 others (2009) Evolution of the texture along the EPICA Dome C ice core. Low Temp. Sci., 68(Suppl.), 91-105

Duval P and Castelnau O (1995) Dynamic recrystallization of ice in polar ice sheets. J. Phys. IV [Paris], 5(C3), 197-205 (doi: 10.1051/jp4:1995317)

Faria SH and 6 others (2009) The multiscale structure of Antarctica. Part I: inland ice. Low Temp. Sci., 68(Suppl.), 39-59 (doi: 10013/epic.35759.d001)

Faria SH, Weikusat I and Azuma N (2014a) The microstructure of polar ice. Part I: Highlights from ice core research. J. Struct. Geol., 61, 2-20 (doi: 10.1016/j.jsg.2013.09.010)

Faria SH, Weikusat I and Azuma N (2014b) The microstructure of polar ice. Part II: State of the art. J. Struct. Geol., 61, 21-49 (doi: 10.1016/j.jsg.2013.11.003) 
Fujita S, Okuyama J, Hori A and Hondoh T (2009) Metamorphism of stratified firn at Dome Fuji, Antarctica: a mechanism for local insolation modulation of gas transport conditions during bubble close off. J. Geophys. Res., 114(F3), F03023, 1-21 (doi: 10.1029/2008JF001143)

Gagliardini O, Durand G and Wang Y (2004) Grain area as a statistical weight for polycrystal constituents. J. Glaciol., 50(168), 87-95 (doi: 10.3189/172756504781830349)

Glen JW (1955) The creep of polycrystalline ice. Proc. R. Soc. London, Ser. A, 228(1175), 519-538 (doi: 10.1098/ rspa.1955.0066)

Gödert G and Hutter K (1998) Induced anisotropy in large ice shields: theory and its homogenization. Contin. Mech. Thermodyn., 10(5), 293-318

Gow AJ and Meese D (2007) Physical properties, crystalline textures and c-axis fabrics of the Siple Dome (Antarctica) ice core. J. Glaciol., 53(183), 573-584 (doi: 10.3189/ $002214307784409252)$

Gow AJ and 6 others (1997) Physical and structural properties of the Greenland Ice Sheet Project 2 ice core : a review. J. Geophys. Res., 102(C12), 26 559-26 575 (doi: 10.1029/97JC00165)

Gusmeroli A, Pettit EC, Kennedy JH and Ritz C (2012) The crystalline fabric of glacial ice from full-waveform borehole sonic logging. J. Geophys. Res., 117(F3), F03021, 1-13 (doi: 10.1029/2012JF002343)

Jacka TH and Jun L (1994) The steady-state crystal size of deforming ice. Ann. Glaciol., 20, 13-18

Kamb WB (1959) Ice petrofabric observation from Blue Glacier, Washington, in relation to theory and experiment. J. Geophys. Res., 64(11), 1891-1909 (doi: 10.1029/JZ064i011p01891)

Kennedy JH, Pettit EC and Di Prinzio CL (2013) The evolution of crystal fabric in ice sheets and its link to climate history. J. Glaciol., 59(214), 357-373 (doi: 10.3189/2013JoG12J159)

Ketcham WM and Hobbs PV (1969) An experimental determination of the surface energies of ice. Philos. Mag., 19(162), 1161-1173 (doi: 10.1080/14786436908228641)

Kipfstuhl S and 6 others (2006) Microstructure mapping: a new method for imaging deformation-induced microstructural features of ice on the grain scale. J. Glaciol., 52(178), 398-406 (doi: 10.3189/172756506781828647)

Kipfstuhl S and 8 others (2009) Evidence of dynamic recrystallization in polar firn. J. Geophys. Res., 114(B5), 1-10 (doi: 10.1029/ 2008JB005583)

Miguel M-C, Vespignani A, Zapperi S, Weiss J and Grasso J-R (2001) Intermittent dislocation flow in viscoplastic deformation. Nature, 410(6829), 667-671 (doi: 10.1038/35070524)

Mohamed G and Bacroix B (2000) Role of stored energy in static recrystallization of cold rolled copper single and multicrystals. Acta Mater., 48(13), 3295-3302

Montagnat M and Duval P (2000) Rate controlling processes in the creep of polar ice: influence of grain boundary migration associated with recrystallization. Earth Planet. Sci. Lett., 183, 179-186 (doi: 10.1016/S0012-821X(00)00262-4)

Montagnat M, Durand G and Duval P (2009) Recrystallization processes in granular ice. Low Temp. Sci., 68(Suppl.), 81-90
Montagnat M and 6 others (2012) Measurements and numerical simulation of fabric evolution along the Talos Dome ice core, Antarctica. Earth Planet. Sci. Lett., 357-358, 168-178 (doi: 10.1016/j.epsl.2012.09.025)

Montagnat $M$ and 11 others (2014) Multiscale modeling of ice deformation behavior. J. Struct. Geol., 68, 78-108 (doi: 10.1016/j.jsg.2013.05.002)

Nelson J and Knight C (1998) Snow crystal habit changes explained by layer nucleation. J. Atmos. Sci., 55(8), 1452-1465 (doi: 10. 1175/1520-0469(1998)055\%3C1452:SCHCEB\%3E2.0.CO;2)

Obbard R, Baker I and Sieg K (2006) Using electron backscatter diffraction patterns to examine recrystallization in polar ice sheets. J. Glaciol., 52(179), 546-557 (doi: 10.3189/ 172756506781828458)

Paterson WSB (1991) Why ice-age ice is sometimes 'soft'. Cold Reg. Sci. Technol., 20, 75-98 (doi: 10.1016/0165-232X(91) 90058-O)

Pettit EC and Waddington ED (2003) Ice flow at low deviatoric stress. J. Glaciol., 49(166), 359-369 (doi: 10.3189/ $172756503781830584)$

Pettit EC, Thorsteinsson T, Jacobson PH and Waddington ED (2007) The role of crystal fabric in flow near an ice divide. J. Glaciol., 53(181), 277-288 (doi: 10.3189/172756507782202766)

Pettit EC and 6 others (2011) The crossover stress, anisotropy and the flow law at Siple Dome, West Antarctica. J. Glaciol., 57(201), 39-52 (doi: 10.3189/002214311795306619)

Shimizu I (2008) Theories and applicability of grain size piezometers: the role of dynamic recrystallization mechanisms. J. Struct. Geol., 30(7), 899-917 (doi: 10.1016/j.jsg.2008.03.004)

Thorsteinsson T (2001) An analytical approach to deformation of anisotropic ice-crystal aggregates. J. Glaciol., 47(158), 507-516 (doi: 10.3189/172756501781832124)

Thorsteinsson T (2002) Fabric development with nearest-neighbor interaction and dynamic recrystallization. J. Geophys. Res., 107(B1), 1-13 (doi: 10.1029/2001JB000244)

Thorsteinsson T, Waddington ED, Taylor KC, Alley RB and Blankenship DD (1999) Strain rate enhancement at Dye 3, Greenland. J. Glaciol., 45(150), 338-345 (doi: 10.3189/ 002214399793377185)

Wang $\mathrm{Y}$, Kipfstuhl S, Azuma N, Thorsteinsson $\mathrm{T}$ and Miller $\mathrm{H}$ (2003) Ice-fabrics study in the upper $1500 \mathrm{~m}$ of the Dome C (East Antarctica) deep ice core. Ann. Glaciol., 37(1), 97-104 (doi: 10.3189/172756403781816031)

Weikusat I, Miyamoto A, Faria H, Kipfstuhl S, Azuma N and Hondoh $\mathrm{T}$ (2011) Subgrain boundaries in Antarctic ice quantified by X-ray Laue diffraction. J. Glaciol., 57(201), 111-120 (doi: 10.3189/ 002214311795306628)

Wilson CJL, Peternell M, Piazolo S and Luzin V (2014) Microstructure and fabric development in ice: lessons learned from in situ experiments and implications for understanding rock evolution. J. Struct. Geol., 61, 50-77 (doi: 10.1016/j.jsg.2013. 05.006)

Woodcock NH (1977) Specification of fabric shapes using an eigenvalue method. J. Struct. Geol., 88, 1231-1236 (doi: 10.1130/0016-7606(1977)88<1231:SOFSUA >2.0.CO;2) 